\title{
Structure coefficients of the Hecke algebra of $\left(\mathcal{S}_{2 n}, \mathcal{B}_{n}\right)$
}

\author{
Omar Tout* \\ LaBRI \\ Université de Bordeaux \\ Talence, France \\ omar.tout@labri.fr
}

Submitted: Jul 22, 2013; Accepted: Nov 5, 2014; Published: Nov 13, 2014

Mathematics Subject Classifications: 05E15

\begin{abstract}
The Hecke algebra of the pair $\left(\mathcal{S}_{2 n}, \mathcal{B}_{n}\right)$, where $\mathcal{B}_{n}$ is the hyperoctahedral subgroup of $\mathcal{S}_{2 n}$, was introduced by James in 1961. It is a natural analogue of the center of the symmetric group algebra. In this paper, we give a polynomiality property of its structure coefficients. Our main tool is a combinatorial algebra which projects onto the Hecke algebra of $\left(\mathcal{S}_{2 n}, \mathcal{B}_{n}\right)$ for every $n$. To build it, by using partial bijections we introduce and study a new class of finite dimensional algebras.
\end{abstract}

Keywords: Hecke algebra of $\left(\mathcal{S}_{2 n}, \mathcal{B}_{n}\right)$, partial bijections, structure coefficients

\section{Introduction}

For a positive integer $n$, let $\mathcal{S}_{n}$ denote the symmetric group of permutations on the set $[n]:=\{1,2, \cdots, n\}$, and let $\mathbb{C}\left[\mathcal{S}_{n}\right]$ denote the group-algebra of $\mathcal{S}_{n}$ over $\mathbb{C}^{1}$, the field of complex numbers. The center of $\mathbb{C}\left[\mathcal{S}_{n}\right]$, denoted by $Z\left(\mathbb{C}\left[\mathcal{S}_{n}\right]\right)$ is a classical object in combinatorics. It is linearly generated by elements $\mathcal{Z}_{\lambda}$, indexed by partitions of $n$, where $\mathcal{Z}_{\lambda}$ is the sum of permutations of $[n]$ with cycle-type $\lambda$. The structure coefficients $c_{\lambda \delta}^{\rho}$ describe the product in this algebra, they are defined by the equation:

$$
\mathcal{Z}_{\lambda} \mathcal{Z}_{\delta}=\sum_{\rho \text { partition of } n} c_{\lambda \delta}^{\rho} \mathcal{Z}_{\rho}
$$

*Partially supported by ANR grant PSYCO ANR-11-JS02-001.

${ }^{1}$ Though all our results remain true in the field $\mathbb{Q}$ of rational numbers, it is usual to consider group algebras over $\mathbb{C}$. 
In other words, $c_{\lambda \delta}^{\rho}$ counts the number of pairs of permutations $(x, y)$ with cycle-type $\lambda$ and $\delta$ such that $x \cdot y=z$ for a fixed permutation $z$ with cycle-type $\rho$. It is known that these coefficients count the number of embeddings of certain graphs into orientable surfaces (see [Cor75]). One of the tools used to calculate these coefficients is the representation theory of the symmetric group, see [JV90, Lemma 3.3]. In [GS98, Theorem 2.1], Goupil and Schaeffer have a formula for $c_{\lambda \delta}^{\rho}$ if one of the partitions $\lambda, \delta$ and $\rho$ is equal to $(n)$. There are no formulas for $c_{\lambda \delta}^{\rho}$ in general.

In 1958, Farahat and Higman proved the polynomiality of the coefficients $c_{\lambda \delta}^{\rho}$ in $n$ when $\lambda, \delta$ and $\rho$ are fixed partitions, completed with parts equal to 1 to get partitions of $n$, [FH59, Theorem 2.2]. More recently, in [IK99], by using objects called partial permutations, the same result is obtained by Ivanov and Kerov. This more recent proof provides a combinatorial description of the coefficients of the relevant polynomials.

Here, we consider the Hecke algebra of the pair $\left(\mathcal{S}_{2 n}, \mathcal{B}_{n}\right)$, denoted by $\mathbb{C}\left[\mathcal{B}_{n} \backslash \mathcal{S}_{2 n} / \mathcal{B}_{n}\right]$, where $\mathcal{B}_{n}$ is the hyperoctahedral group. It was introduced by James in [Jam61] and it also has a basis indexed by partitions of $n$. The algebra $\mathbb{C}\left[\mathcal{B}_{n} \backslash \mathcal{S}_{2 n} / \mathcal{B}_{n}\right]$ is a natural analogue of $Z\left(\mathbb{C}\left[\mathcal{S}_{n}\right]\right)$ for several reasons. Goulden and Jackson proved in [GJ96] that its structure coefficients count graphs drawn on non-oriented surfaces. To get formulas for these coefficients, zonal characters are used instead of irreducible characters of the symmetric group, see [Mac95, Section VII, 2].

In this paper we establish a polynomiality property for the structure coefficients of the Hecke algebra of $\left(\mathcal{S}_{2 n}, \mathcal{B}_{n}\right)$. Namely, we prove that these coefficients can be written as the product of the number $2^{n} n$ ! with a polynomial in $n$. In some specific basis, this polynomial has non-negative coefficients that have a combinatorial interpretation. Moreover, we are able to give an upper bound for its degree. Our proof is based on the construction of a universal algebra which projects onto the Hecke algebra of $\left(\mathcal{S}_{2 n}, \mathcal{B}_{n}\right)$ for every $n^{2}$. This method was already used by Ivanov and Kerov in [IK99]. What is original in our approach is that the product in our universal algebra is computed as an average of combinatorial objects called partial bijections of $[2 n]$. Recently, P.-L. Méliot has used this same idea in [Mél13] to give a polynomiality property for the structure coefficients of the center of the group-algebra $\mathbb{C}\left[G L\left(n, \mathbb{F}_{q}\right)\right]$, where $G L\left(n, \mathbb{F}_{q}\right)$ is the group of invertible $n \times n$ matrices with coefficients in $\mathbb{F}_{q}$. Because of the similarities between Meliot's construction and ours, we are convinced we should build a general framework in which such a result (polynomiality of the structure coefficients) always holds. This is the subject of future work.

A weaker version of our polynomiality result (without non-negativity of the coefficients) for the structure coefficients of Hecke algebra of $\left(\mathcal{S}_{2 n}, \mathcal{B}_{n}\right)$ has been established by an indirect approach using Jack polynomials in [DF14, Proposition 5.3]. There is no combinatorial description in that proof. By a different approach than ours, in [AC12], Aker and Can study the Hecke ring $\mathbb{C}\left[\mathcal{B}_{n} \backslash \mathcal{S}_{2 n} / \mathcal{B}_{n}\right]$, however, it seems that there is a minor issue in their proof of polynomiality of the structure coefficients $([\mathrm{Can} 13])^{3}$. A universal

\footnotetext{
${ }^{2}$ In this sense, we shall call it a universal algebra.

${ }^{3}$ Recently, Can and Özden published a paper, see [BÖ14], in which they suggested a correction to the Can and Aker's proof of polynomiality of the structure coefficients of the Hecke ring $\mathbb{C}\left[\mathcal{B}_{n} \backslash \mathcal{S}_{2 n} / \mathcal{B}_{n}\right]$ given
} 
algebra also appears in this paper, but it does not have a combinatorial realization as ours.

As it is mentioned earlier, our proof goes through the construction of a universal algebra which projects onto the Hecke algebra of $\left(\mathcal{S}_{2 n}, \mathcal{B}_{n}\right)$ for every $n$. We are able to give a link between this algebra, and the algebra of shifted symmetric functions. Shifted symmetric functions have been introduced and studied by A. Okounkov and G. Olshansky in 1996, see [OO97]. They are deformations of usual symmetric functions that display remarkable properties.

The paper is organized as follows. In Section 2, we review all necessary definitions to describe the Hecke algebra of $\left(\mathcal{S}_{2 n}, \mathcal{B}_{n}\right)$. Then, we state our main result about its structure coefficients. We start Section 3 by introducing partial bijections of $[2 n]$ then we build our universal algebra. We use this algebra in Section 4 to prove our main result, Theorem 2.1. In Section 5, we show how the universal algebra is related with the algebra of shifted symmetric functions and in Section 6 we exhibit some filtrations on this universal algebra, which implies the above mentioned upper bounds for the degree of the polynomials.

\section{Definitions and statement of the main result}

As usual, we denote by $\mathbb{N}$ the set of non-negative integers and by $\mathbb{N}^{*}$ the set of positive integers.

\subsection{Partitions}

Since partitions index bases of the algebras studied in this paper, we recall the main definitions. A partition $\lambda$ is a list of integers $\left(\lambda_{1}, \ldots, \lambda_{l}\right)$ where $\lambda_{1} \geqslant \lambda_{2} \geqslant \ldots \lambda_{l} \geqslant 1$. The $\lambda_{i}$ are called the parts of $\lambda$; the size of $\lambda$, denoted by $|\lambda|$, is the sum of all of its parts. If $|\lambda|=n$, we say that $\lambda$ is a partition of $n$ and we will write $\lambda \vdash n$. The number of parts of $\lambda$ is denoted by $l(\lambda)$. We will also use the exponential notation $\lambda=\left(1^{m_{1}(\lambda)}, 2^{m_{2}(\lambda)}, 3^{m_{3}(\lambda)}, \ldots\right)$, where $m_{i}(\lambda)$ is the number of parts equal to $i$ in the partition $\lambda$. If $\lambda$ and $\delta$ are two partitions we define the union $\lambda \cup \delta$ as the following partition:

$$
\lambda \cup \delta=\left(1^{m_{1}(\lambda)+m_{1}(\delta)}, 2^{m_{2}(\lambda)+m_{2}(\delta)}, 3^{m_{3}(\lambda)+m_{3}(\delta)}, \ldots\right) .
$$

A partition is called proper if it does not have any part equal to 1 . The proper partition associated to a partition $\lambda$ is the partition $\bar{\lambda}:=\lambda \backslash\left(1^{m_{1}(\lambda)}\right)=\left(2^{m_{2}(\lambda)}, 3^{m_{3}(\lambda)}, \ldots\right)$.

\subsection{Permutations and Coset type}

For a permutation $\omega$, we use the one-line notation $\omega_{1} \omega_{2} \cdots \omega_{n}$, where $\omega_{i}=\omega(i)$. The set $\mathcal{S}_{n}$ of all permutations of $[n]$ is a group for the composition called the symmetric group of size $n$.

in $[\mathrm{AC} 12]$. 
To each permutation $\omega$ of $2 n$ we associate a graph $\Gamma(\omega)$ with $2 n$ vertices located on a circle. Each vertex is labelled by two labels (exterior and interior). The exterior labels run through natural numbers from 1 to $2 n$ around the circle. The interior label of the vertex with exterior label $i$ is $\omega(i)$. We link the vertices with exterior (resp. interior) labels $2 i-1$ and $2 i$ by an exterior (resp. interior) edge. As every vertex has degree 2 , the graph $\Gamma(\omega)$ is a disjoint union of cycles. Since exterior and interior edges alternate, all cycles have even lengths $2 \lambda_{1} \geqslant 2 \lambda_{2} \geqslant 2 \lambda_{3} \geqslant \cdots$. The coset-type of $\omega$, denoted by $\operatorname{ct}(\omega)$, is the partition $\left(\lambda_{1}, \lambda_{2}, \lambda_{3}, \ldots\right)$ of $n$.

Example 2.1. The graph $\Gamma(\omega)$ associated to the permutation $\omega=24931105867 \in \mathcal{S}_{10}$ is drawn on Figure 1. It has two cycles of length 6 and 4 , so $\operatorname{ct}(\omega)=(3,2)$.

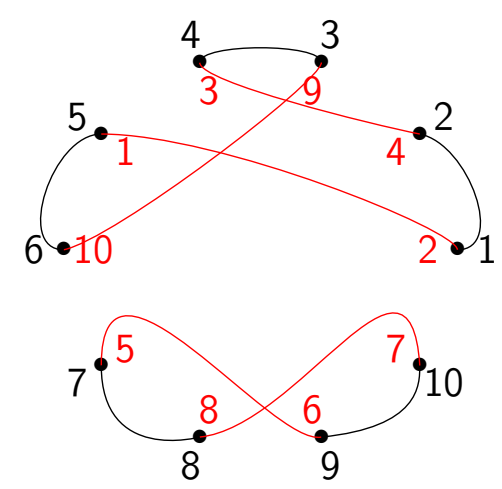

Figure 1: The graph $\Gamma(\omega)$ from Example 2.1.

For every $k \geqslant 1$, we set $\rho(k):=\{2 k-1,2 k\}$. The hyperoctahedral group $\mathcal{B}_{n}$ is the subgroup of $\mathcal{S}_{2 n}$ of permutations $\omega$ such that, for every $1 \leqslant k \leqslant n$, there exists $1 \leqslant k^{\prime} \leqslant n$ with $\omega(\rho(k))=\rho\left(k^{\prime}\right)$. In other words $\mathcal{B}_{n}=\left\{\omega \in \mathcal{S}_{2 n} \mid c t(\omega)=\left(1^{n}\right)\right\}$. For example, $431265 \in \mathcal{B}_{3}$.

A $\mathcal{B}_{n}$-double coset of $\mathcal{S}_{2 n}$ is the set $\mathcal{B}_{n} x \mathcal{B}_{n}=\left\{b x b^{\prime} ; b, b^{\prime} \in \mathcal{B}_{n}\right\}$ for some $x \in \mathcal{S}_{2 n}$. It is known, see [Mac95, page 401], that two permutations of $\mathcal{S}_{2 n}$ are in the same $\mathcal{B}_{n}$-double coset if and only if they have the same coset-type. Thus, if $x \in \mathcal{S}_{2 n}$ has coset-type $\lambda$, we have:

$$
\mathcal{B}_{n} x \mathcal{B}_{n}=\left\{y \in \mathcal{S}_{2 n} \text { such that } \operatorname{ct}(y)=\lambda\right\} .
$$

\subsection{The Hecke algebra of $\left(\mathcal{S}_{2 n}, \mathcal{B}_{n}\right)$}

The symmetric group algebra of $n$, denoted by $\mathbb{C}\left[\mathcal{S}_{n}\right]$, is the algebra over $\mathbb{C}$ linearly generated by all permutations of $[n]$. The group $\mathcal{B}_{n} \times \mathcal{B}_{n}$ acts on $\mathbb{C}\left[\mathcal{S}_{2 n}\right]$ by the following action: $\left(b, b^{\prime}\right) \cdot x=b x b^{-1}$, called the $\mathcal{B}_{n} \times \mathcal{B}_{n}$-action. The Hecke algebra of $\left(\mathcal{S}_{2 n}, \mathcal{B}_{n}\right)$, denoted by $\mathbb{C}\left[\mathcal{B}_{n} \backslash \mathcal{S}_{2 n} / \mathcal{B}_{n}\right]$, is the sub-algebra of $\mathbb{C}\left[\mathcal{S}_{2 n}\right]$ of elements invariant under the $\mathcal{B}_{n} \times \mathcal{B}_{n}$-action. Recall that $\mathcal{B}_{n}$-double cosets are indexed by partitions of $n$. Here, we index the basis by proper partitions of size less or equal than $n$, which are in bijection 
with partitions of $n$. Therefore, the set

$$
\left\{K_{\lambda}(n): \lambda \text { is a proper partition with }|\lambda| \leqslant n\right\}
$$

forms a basis for $\mathbb{C}\left[\mathcal{B}_{n} \backslash \mathcal{S}_{2 n} / \mathcal{B}_{n}\right]$, where $K_{\lambda}(n)$ is the sum of all permutations from $\mathcal{S}_{2 n}$ with coset-type $\lambda \cup\left(1^{n-|\lambda|}\right)$. So, for any two proper partitions $\lambda$ and $\delta$ with size at most $n$, there exist complex numbers $\alpha_{\lambda \delta}^{\rho}(n)$ such that:

$$
K_{\lambda}(n) \cdot K_{\delta}(n)=\sum_{\substack{\rho \text { proper partition } \\|\rho| \leqslant n}} \alpha_{\lambda \delta}^{\rho}(n) K_{\rho}(n)
$$

\subsection{Main result}

In this paper, we obtain a polynomiality property for the structure coefficients $\alpha_{\lambda \delta}^{\rho}(n)$ of the Hecke algebra of $\left(\mathcal{S}_{2 n}, \mathcal{B}_{n}\right)$. More precisely, we prove the following theorem. We will use the standard notation $(n)_{k}:=\frac{n !}{(n-k) !}=n(n-1) \cdots(n-k+1)$ for $0 \leqslant k \leqslant n$.

Theorem 2.1. Let $\lambda, \delta$ and $\rho$ be three proper partitions. Than we have:

$$
\alpha_{\lambda \delta}^{\rho}(n)= \begin{cases}2^{n} n ! f_{\lambda \delta}^{\rho}(n) & \text { if } \quad n \geqslant|\rho|, \\ 0 & \text { if } n<|\rho|,\end{cases}
$$

where $f_{\lambda \delta}^{\rho}(n)=\sum_{j=0}^{|\lambda|+|\delta|-|\rho|} a_{j}(n-|\rho|)_{j}$ is a polynomial in $n$ and the $a_{j}$ 's are non-negative rational numbers.

Example 2.2. Let us compute the structure coefficient $\alpha_{(2)(2)}^{\emptyset}(n)$. We have:

$$
K_{(2)}(n)=\sum_{\substack{\omega \in \mathcal{S}_{2 n} \\ c t(\omega)=(2) \cup\left(1^{n-2}\right)}} \omega .
$$

To find the coefficient of $K_{\emptyset}(n)$ in $K_{(2)}(n) \cdot K_{(2)}(n)$, we fix a permutation with coset-type $\left(1^{n}\right)$, for example $\operatorname{Id}_{2 n}$, and we look in how many ways we can obtain $\operatorname{Id}_{2 n}$ as a product of two elements $\sigma \cdot \beta$ where $\operatorname{ct}(\sigma)=\operatorname{ct}(\beta)=\left(2,1^{n-2}\right)$. Thus we are looking for the number of permutations $\sigma \in \mathcal{S}_{2 n}$ such that $\operatorname{ct}(\sigma)=\operatorname{ct}\left(\sigma^{-1}\right)=\left(2,1^{n-2}\right)$. But, for any $\sigma \in \mathcal{S}_{2 n}$ with $c t(\sigma)=\left(2,1^{n-2}\right)$, its inverse has the same coset-type. Therefore $\alpha_{(2)(2)}^{\emptyset}(n)$ is the number of permutations of coset-type $\left(2,1^{n-2}\right)$, which is

$$
\frac{\left(2^{n} n !\right)^{2}}{2^{n-1}(2 \cdot(n-2) !)}=n(n-1) 2^{n} n !
$$

by [Mac95, page 402]. 


\subsection{Major steps of the proof}

The idea of the proof is to build a universal algebra $\mathcal{A}_{\infty}$ over $\mathbb{C}$ satisfying the following properties:

1. For every $n \in \mathbb{N}^{*}$, there exists a morphism of algebras $\theta_{n}: \mathcal{A}_{\infty} \longrightarrow \mathbb{C}\left[\mathcal{B}_{n} \backslash \mathcal{S}_{2 n} / \mathcal{B}_{n}\right]$.

2. Every element $x$ in $\mathcal{A}_{\infty}$ is written in a unique way as an infinite linear combination of elements $T_{\lambda}$, indexed by partitions. This implies that, for any two partitions $\lambda$ and $\delta$, there exist non-negative rational numbers $b_{\lambda \delta}^{\rho}$ such that:

$$
T_{\lambda} * T_{\delta}=\sum_{\rho \text { partition }} b_{\lambda \delta}^{\rho} T_{\rho}
$$

3. The morphism $\theta_{n}$ sends $T_{\lambda}$ to a multiple of $K_{\bar{\lambda}}(n)$.

To build $\mathcal{A}_{\infty}$, we use combinatorial objects called "partial bijections". For every $n \in \mathbb{N}^{*}$, we construct an algebra $\mathcal{A}_{n}$ using the set of partial bijections of $[2 n]$. The algebra $\mathcal{A}_{\infty}$ is defined as the projective limit of this sequence $\left(\mathcal{A}_{n}\right)$.

The projection $p_{n}: \mathcal{A}_{\infty} \rightarrow \mathcal{A}_{n}$ involves coefficients which are polynomials in $n$. By defining the extension of a partial bijection of $[2 n]$ to the set $[2 n]$, we construct a morphism from $\mathcal{A}_{n}$ to $\mathbb{C}\left[\mathcal{B}_{n} \backslash \mathcal{S}_{2 n} / \mathcal{B}_{n}\right]$. Its coefficients involve the number $2^{n} n$ !. It turns out that the morphism $\theta_{n}$ is a composition of morphisms as shown in Figure 2. The final step consists

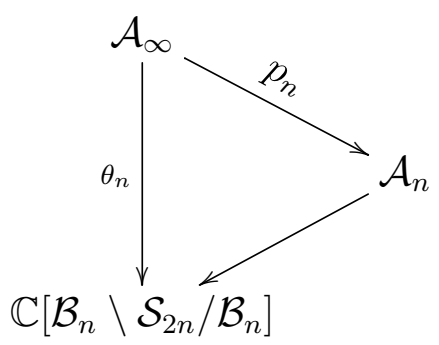

Figure 2: Diagram of essential algebras and morphisms for our proof.

of applying the chain of morphisms in Figure 2 to equation (2).

\section{$3 \quad$ The partial bijection algebra}

In this section we define the set of partial bijections of $[2 n]$. With this set, we build the algebras and homomorphisms that appear in Figure 2.

\subsection{Definition}

We start by defining partial bijections of $[2 n]$ and the partial bijection algebra. Then, we introduce the notion of trivial extension of a partial bijection of $[2 n]$ and we use it 
to build a homomorphism between the partial bijection algebra of $n$ and the symmetric group algebra of $2 n$.

For $n \in \mathbb{N}^{*}$, we define $\mathbf{P}_{n}$ to be the following set:

$$
\mathbf{P}_{n}:=\left\{\rho\left(k_{1}\right) \cup \cdots \cup \rho\left(k_{i}\right) \mid 1 \leqslant i \leqslant n, 1 \leqslant k_{1}<\cdots<k_{i} \leqslant n\right\} .
$$

Definition 3.1. A partial bijection of $[2 n]$ is a triple $\left(\sigma, d, d^{\prime}\right)$ where $d, d^{\prime} \in \mathbf{P}_{n}$ and $\sigma$ : $d \longrightarrow d^{\prime}$ is a bijection. The set $d$ is the domain of $\left(\sigma, d, d^{\prime}\right)$ while $d^{\prime}$ is its co-domain. We denote by $Q_{n}$ the set of all partial bijections of $[2 n]$.

Remark 3.2. In semigroup theory, the term "partial bijection" refers to an arbitrary injection from a subset $A$ of $[n]$ to $[n]$. From this point of view, a partial bijection of $[2 n]$ is a very special partial bijection in semigroup theory. However, we choose this notation as a similar, in the case of the Hecke algebra of $\left(\mathcal{S}_{2 n}, \mathcal{B}_{n}\right)$, to the notion of "partial permutation" used by Ivanov and Kerov in [IK99]. This notation will not cause any confusion since the notion of partial bijection in semigroup theory is not used at all in this paper.

Remark 3.3. For any positive integer $n$, let $R_{n}$ be the set of all one-to-one maps $f$ : $d(f) \longrightarrow c(f)$ where $c(f), d(f) \subseteq[n]$. The set $R_{n}$ with the composition of maps is a monoid - that is the composition is associative and $R_{n}$ has an identity element - called the symmetric inverse semigroup. With this composition, the set of partial bijections $Q_{n}$ forms a submonoid of $R_{2 n}$. It is known, see [Sol90], that $R_{2 n}$ is in bijection with the rook monoid $\mathcal{R}_{2 n}$. It is important to notice that this obvious structure on $Q_{n}$ does not enter the picture in here. The useful product in this work will be defined later in this section.

It should be clear that

$$
\left|Q_{n}\right|=\sum_{k=0}^{n}\left(\begin{array}{l}
n \\
k
\end{array}\right)^{2}(2 k) !
$$

A permutation $\sigma$ of $2 n$ can be written as $(\sigma,[2 n],[2 n])$, so the set $\mathcal{S}_{2 n}$ can be considered as a subset of $Q_{n}$.

Notation. For any partial bijection $\alpha$, we will use the convention that $\sigma$ (resp. $d, d^{\prime}$ ) is the first (resp. second, third) element of the triple defining $\alpha$. The same convention holds for $\widetilde{\alpha}, \alpha_{i}, \hat{\alpha} \ldots$

Observation 3.2. In the same way as in Section 2.2, we can associate to each partial bijection $\alpha$ of $[2 n]$ a graph $\Gamma(\alpha)$ with $|d|$ vertices placed on a circle. The exterior (resp. interior) labels are the elements of the set $d$ (resp. $d^{\prime}$ ). Since the sets $d$ and $d^{\prime}$ are in $\mathbf{P}_{n}$, we can link $2 i$ with $2 i-1$ as in the case $d=d^{\prime}=[2 n]$. So, the definition of coset-type extends naturally to partial bijections. We denote by $\operatorname{ct}(\alpha)$ or $\operatorname{ct}(\sigma)$ the coset-type of a partial bijection $\alpha$.

Example 3.1. Let $\alpha=\left(\sigma, d, d^{\prime}\right)$ be the partial bijection of [16] where

$$
d=\{3,4,5,6,9,10,11,12,13,14\}, d^{\prime}=\{1,2,3,4,7,8,9,10,15,16\}
$$


and $\sigma$ is given by the following two-lines notation:

$$
\sigma=\begin{array}{cccccccccc}
3 & 4 & 5 & 6 & 9 & 10 & 11 & 12 & 13 & 14 \\
9 & 16 & 1 & 15 & 10 & 2 & 4 & 8 & 3 & 7
\end{array}
$$

which means that $\sigma(3)=9, \sigma(4)=1$ and so on. The graph $\Gamma(\alpha)$ is drawn on Figure 3 . It has two cycles of length 6 and 4 , so $\operatorname{ct}(\alpha)=(3,2)$.

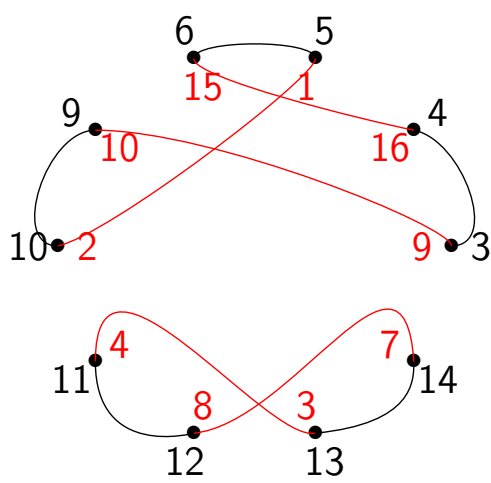

Figure 3: The graph $\Gamma(\alpha)$.

Definition 3.4. Let $\left(\sigma, d, d^{\prime}\right)$ and $\left(\widetilde{\sigma}, \widetilde{d}, \widetilde{d}^{\prime}\right)$ be two partial bijections of $[2 n]$. We say that $\left(\widetilde{\sigma}, \widetilde{d}, \widetilde{d}^{\prime}\right)$ is a trivial extension of $\left(\sigma, d, d^{\prime}\right)$ if:

$$
d \subseteq \widetilde{d}, \widetilde{\sigma}_{\left.\right|_{d}}=\sigma \text { and } \operatorname{ct}(\widetilde{\sigma})=\operatorname{ct}(\sigma) \cup\left(1^{\frac{|\widetilde{d} \backslash d|}{2}}\right) .
$$

We denote by $P_{\alpha}(n)$ the set of all trivial extensions of $\alpha$ in $Q_{n}$.

Example 3.2. Let $\alpha$ be the partial bijection of [16] given in Example 3.1. Let $\widetilde{d}=d \cup$ $\{1,2,15,16\}, \widetilde{d^{\prime}}=d^{\prime} \cup\{5,6,11,12\}$ and consider the following bijection:

$$
\widetilde{\sigma}=\begin{array}{cccccccccccccc}
\mathbf{1} & \mathbf{2} & 3 & 4 & 5 & 6 & 9 & 10 & 11 & 12 & 13 & 14 & \mathbf{1 5} & \mathbf{1 6} \\
\mathbf{1 2} & \mathbf{1 1} & 9 & 16 & 1 & 15 & 10 & 2 & 4 & 8 & 3 & 7 & \mathbf{5} & \mathbf{6}
\end{array} .
$$

Then, $\widetilde{\alpha}=\left(\widetilde{\sigma}, \widetilde{d}, \widetilde{d^{\prime}}\right)$ is a trivial extension of $\alpha$. In the same way $\hat{\alpha}=\left(\hat{\sigma}, \hat{d}, \hat{d}^{\prime}\right)$, where $\hat{d}=\hat{d}^{\prime}=[16]$ and

$$
\hat{\sigma}=\begin{array}{cccccccccccccccc}
\mathbf{1} & \mathbf{2} & 3 & 4 & 5 & 6 & \mathbf{7} & \mathbf{8} & 9 & 10 & 11 & 12 & 13 & 14 & \mathbf{1 5} & \mathbf{1 6} \\
\mathbf{1 3} & \mathbf{1 4} & 9 & 16 & 1 & 15 & \mathbf{1 2} & \mathbf{1 1} & 10 & 2 & 4 & 8 & 3 & 7 & \mathbf{6} & \mathbf{5}
\end{array},
$$

is also a trivial extension of $\alpha$.

Lemma 3.1. Let $\alpha$ be a partial bijection of $[2 n]$ and $X$ an element of $\mathbf{P}_{n}$ such that $d \subseteq X$. The number of trivial extensions $\widetilde{\alpha}$ of $\alpha$ such that $\widetilde{d}=X$ is

$$
(2 n-|d|) \cdot(2 n-|d|-2) \cdots(2 n-|d|-|X \backslash d|+2)=2^{\frac{|X \backslash d|}{2}}\left(n-\frac{|d|}{2}\right)_{\frac{|X \backslash d|}{2}} .
$$

We have the same formula for the number of trivial extensions $\widetilde{\alpha}$ such that $\widetilde{d^{\prime}}=X$. 
Proof. Straightforward by induction.

Consider $\mathcal{D}_{n}=\mathbb{C}\left[Q_{n}\right]$ the vector space with basis $Q_{n}$. We want to endow it with an algebra structure. Let $\alpha_{1}$ and $\alpha_{2}$ be two partial bijections. If $d_{1}=d_{2}^{\prime}$, we can compose $\alpha_{1}$ and $\alpha_{2}$ and we define $\alpha_{1} * \alpha_{2}=\alpha_{1} \circ \alpha_{2}=\left(\sigma_{1} \circ \sigma_{2}, d_{2}, d_{1}^{\prime}\right)$. Otherwise, we need to extend $\alpha_{1}$ and $\alpha_{2}$ to partial bijections $\widetilde{\alpha_{1}}$ and $\widetilde{\alpha_{2}}$ such that $\widetilde{d_{1}}=\widetilde{d_{2}^{\prime}}$. Since there exist several trivial extensions of $\alpha_{1}$ and $\alpha_{2}$, a natural choice is to take the average of the composition of all possible trivial extensions. Let $E_{\alpha_{1}}^{\alpha_{2}}(n)$ be the following set:

$$
E_{\alpha_{1}}^{\alpha_{2}}(n):=\left\{\left(\widetilde{\alpha_{1}}, \widetilde{\alpha_{2}}\right) \in P_{\alpha_{1}}(n) \times P_{\alpha_{2}}(n) \text { such that } \widetilde{d_{1}}=\widetilde{d}_{2}^{\prime}=d_{1} \cup d_{2}^{\prime}\right\} .
$$

Elements of $E_{\alpha_{1}}^{\alpha_{2}}(n)$ are schematically represented on Figure 4.

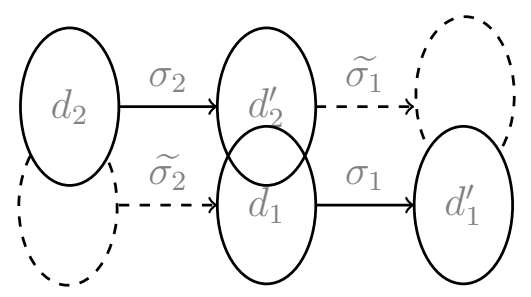

Figure 4: Schematic representation of elements of $E_{\alpha_{1}}^{\alpha_{2}}(n)$.

Convention for figures. Throughout the paper, we will use the following conventions for figures that represent elements of some sets.

- The data defining the set (so fixed when we go from an element of the set to another) is drawn using plain shapes.

- The data variable in the set (thus corresponding to the elements of the set) is drawn using dashed shapes.

To simplify, we will identify a partial bijection $\alpha$ with its bijection $\sigma$ using the two-lines notation. The first line will represent the set $d$ while the second will represent $d^{\prime}$.

Example 3.3. Consider the two partial bijections $\alpha_{1}$ and $\alpha_{2}$ of [6] given below,

$$
\alpha_{1}=\begin{array}{llll}
1 & 2 & 5 & 6 \\
3 & 2 & 1 & 4
\end{array} \text { and } \alpha_{2}=\begin{array}{llll}
3 & 4 & 5 & 6 \\
5 & 6 & 3 & 4
\end{array}
$$

Then, $E_{\alpha_{1}}^{\alpha_{2}}(3)$ is the set of the following four elements.

$$
\begin{aligned}
& \left(\begin{array}{lllllllllllll}
1 & 2 & \mathbf{3} & \mathbf{4} & 5 & 6 & \mathbf{1} & \mathbf{2} & 3 & 4 & 5 & 6 \\
3 & 2 & \mathbf{5} & \mathbf{6} & 1 & 4 & \mathbf{1} & \mathbf{2} & 5 & 6 & 3 & 4
\end{array}\right),\left(\begin{array}{llllllllllll}
1 & 2 & \mathbf{3} & \mathbf{4} & 5 & 6 & \mathbf{1} & \mathbf{2} & 3 & 4 & 5 & 6 \\
3 & 2 & \mathbf{6} & \mathbf{5} & 1 & 4 & \mathbf{1} & \mathbf{2} & 5 & 6 & 3 & 4
\end{array}\right) \\
& \left(\begin{array}{llllllllllll}
1 & 2 & \mathbf{3} & \mathbf{4} & 5 & 6 & \mathbf{1} & \mathbf{2} & 3 & 4 & 5 & 6 \\
3 & 2 & \mathbf{5} & \mathbf{6} & 1 & 4 & \mathbf{2} & \mathbf{1} & 5 & 6 & 3 & 4
\end{array}\right),\left(\begin{array}{llllllllllll}
1 & 2 & \mathbf{3} & \mathbf{4} & 5 & 6 & \mathbf{1} & \mathbf{2} & 3 & 4 & 5 & 6 \\
3 & 2 & \mathbf{6} & \mathbf{5} & 1 & 4 & \mathbf{2} & \mathbf{1} & 5 & 6 & 3 & 4
\end{array}\right)
\end{aligned}
$$


We define the product of $\alpha_{1}$ and $\alpha_{2}$ as follows:

$$
\alpha_{1} * \alpha_{2}:=\frac{1}{\left|E_{\alpha_{1}}^{\alpha_{2}}(n)\right|} \sum_{\left(\widetilde{\alpha_{1}}, \widetilde{\alpha_{2}}\right) \in E_{\alpha_{1}}^{\alpha_{2}}(n)} \widetilde{\alpha_{1}} \circ \widetilde{\alpha_{2}} .
$$

By Lemma 3.1, we have:

$$
\left|E_{\alpha_{1}}^{\alpha_{2}}(n)\right|=2^{\frac{\left|d_{2}^{\prime} \backslash d_{1}\right|}{2}+\frac{\left|d_{1} \backslash d_{2}^{\prime}\right|}{2}} \cdot\left(n-\frac{\left|d_{1}^{\prime}\right|}{2}\right)_{\left(\frac{\left|d_{2}^{\prime} \backslash d_{1}\right|}{2}\right)} \cdot\left(n-\frac{\left|d_{2}\right|}{2}\right)_{\left(\frac{\left|d_{1} \backslash d_{2}^{\prime}\right|}{2}\right)} .
$$

The partial bijections of $[2 n]$ form a basis for $\mathcal{D}_{n}$. We extend the product $*$ by linearity to get a product on $\mathcal{D}_{n}$.

Proposition 3.2. The product $*$ is associative. In other words, $\mathcal{D}_{n}$ is a (non-unital) algebra. Proof. Postponed to the next section.

We will illustrate the associativity by a simple example given below.

Example 3.4. Let $\alpha_{1}$ and $\alpha_{2}$ be the two partial bijections of [6] given in Example 3.3, using the set $E_{\alpha_{1}}^{\alpha_{2}}(3)$, we have:

$$
\begin{aligned}
& \alpha_{1} * \alpha_{2} \\
= & \frac{1}{4}\left[\begin{array}{llllllllllllllllllllllll}
1 & 2 & 3 & 4 & 5 & 6 \\
3 & 2 & 1 & 4 & 5 & 6
\end{array}+\begin{array}{lllllllllllll}
1 & 2 & 3 & 4 & 5 & 6 \\
3 & 2 & 1 & 4 & 6 & 5
\end{array}+\begin{array}{llllllll}
1 & 2 & 3 & 4 & 5 & 6 \\
2 & 3 & 1 & 4 & 5 & 6
\end{array}+\begin{array}{lllllll}
1 & 2 & 3 & 5 & 6 \\
2 & 3 & 1 & 4 & 6 & 5
\end{array}\right] .
\end{aligned}
$$

Take the partial bijection $\alpha_{3}$ of [6] defined by:

$$
\alpha_{3}=\begin{array}{llll}
1 & 2 & 3 & 4 \\
5 & 1 & 6 & 2
\end{array}
$$

In the same way we can verify that:

$$
\begin{aligned}
& \alpha_{2} * \alpha_{3} \\
= & \frac{1}{4}\left[\begin{array}{llllll}
1 & 2 & 3 & 4 & 5 & 6 \\
3 & 1 & 4 & 2 & 5 & 6
\end{array}+\begin{array}{llllllllllllllllll}
1 & 2 & 3 & 4 & 5 & 6 \\
3 & 1 & 4 & 2 & 6 & 5
\end{array}+\begin{array}{lllllllll}
1 & 2 & 3 & 4 & 5 & 6 \\
3 & 2 & 4 & 1 & 5 & 6
\end{array}+\begin{array}{llllll}
1 & 2 & 3 & 4 & 5 & 6 \\
3 & 2 & 4 & 1 & 6 & 5
\end{array}\right],
\end{aligned}
$$

and that:

$$
\begin{aligned}
& \alpha_{1} *\left(\alpha_{2} * \alpha_{3}\right) \\
& =\left(\alpha_{1} * \alpha_{2}\right) * \alpha_{3} \\
& =\frac{1}{8}\left[\begin{array}{llllll}
1 & 2 & 3 & 4 & 5 & 6 \\
5 & 3 & 6 & 2 & 1 & 4
\end{array}+\begin{array}{llllll}
1 & 2 & 3 & 4 & 5 & 6 \\
5 & 3 & 6 & 2 & 4 & 1
\end{array}+\begin{array}{llllll}
1 & 2 & 3 & 4 & 5 & 6 \\
6 & 3 & 5 & 2 & 1 & 4
\end{array}\right] \\
& +\frac{1}{8}\left[\begin{array}{llllll}
1 & 2 & 3 & 4 & 5 & 6 \\
6 & 3 & 5 & 2 & 4 & 1
\end{array}+\begin{array}{cccccc}
1 & 2 & 3 & 4 & 5 & 6 \\
5 & 2 & 6 & 3 & 1 & 4
\end{array}+\begin{array}{llllll}
1 & 2 & 3 & 4 & 5 & 6 \\
5 & 2 & 6 & 3 & 4 & 1
\end{array}\right] \\
& +\frac{1}{8}\left[\begin{array}{llllll}
1 & 2 & 3 & 4 & 5 & 6 \\
6 & 2 & 5 & 3 & 1 & 4
\end{array}+\begin{array}{llllll}
1 & 2 & 3 & 4 & 5 & 6 \\
6 & 2 & 5 & 3 & 4 & 1
\end{array}\right] .
\end{aligned}
$$


Proposition 3.3. The following function extends to an algebra homomorphism between $\mathbb{C}\left[Q_{n}\right]$ and $\mathbb{C}\left[\mathcal{S}_{2 n}\right]$.

$$
\begin{aligned}
\psi_{n}: \mathbb{C}\left[Q_{n}\right] & \longrightarrow \quad \frac{1}{\alpha} \quad \mapsto \quad \frac{\mathbb{C}\left[\mathcal{S}_{2 n}\right]}{2^{n-\frac{|d|}{2}}\left(n-\frac{|d|}{2}\right) !} \sum_{\hat{\alpha} \in \mathcal{S}_{2 n} \cap P_{\alpha}(n)} \hat{\sigma}
\end{aligned}
$$

Proof. Let $\alpha_{1}$ and $\alpha_{2}$ be two basis elements of $\mathbb{C}\left[Q_{n}\right]$. We refer to Figure 4 and denote $2 b=\left|d_{2}\right|=\left|d_{2}^{\prime}\right|, 2 c=\left|d_{1}\right|=\left|d_{1}^{\prime}\right|$ and $2 e=\left|d_{2}^{\prime} \cap d_{1}\right|$. We first prove that:

$$
\begin{aligned}
& \sum_{\widehat{\alpha_{1}} \in \mathcal{S}_{2 n} \cap P_{\alpha_{1}}(n)} \sum_{\widehat{\alpha_{2}} \in \mathcal{S}_{2 n} \cap P_{\alpha_{2}}(n)} \widehat{\sigma_{1}} \circ \widehat{\sigma_{2}} \\
& =2^{n-(b+c-e)}(n-(b+c-e)) ! \sum_{\left(\widetilde{\alpha_{1}}, \widetilde{\alpha_{2}}\right) \in E_{\alpha_{2}}^{\alpha_{1}}(n)} \sum_{\widetilde{\alpha_{1} \circ \widetilde{\alpha_{2}}} \in \mathcal{S}_{2 n} \cap P_{\widetilde{\alpha_{1}} \circ \widetilde{\alpha_{2}}}(n)} \widetilde{\widetilde{\sigma_{1} \circ \widetilde{\sigma_{2}}}} .
\end{aligned}
$$

We fix $\left(\widetilde{\alpha_{1}}, \widetilde{\alpha_{2}}\right) \in E_{\alpha_{1}}^{\alpha_{2}}(n)$ and $\omega \in \mathcal{S}_{2 n} \cap P_{\widetilde{\alpha_{1}} \circ \widetilde{\alpha_{2}}}(n)$, i.e.:

$$
\omega_{\left.\right|_{d_{2}}}=\widetilde{\sigma_{1}} \circ \widetilde{\sigma_{2}} \text { and } \operatorname{ct}(\omega)=\operatorname{ct}\left(\widetilde{\sigma_{1}} \circ \widetilde{\sigma_{2}}\right) \cup\left(1^{(n-(b+c-e))}\right) \text {. }
$$

We look for the number of permutations $\widehat{\sigma_{1}}$ and $\widehat{\sigma_{2}}$ in $\mathcal{S}_{2 n} \cap P_{\alpha_{1}}(n)$ and $\mathcal{S}_{2 n} \cap P_{\alpha_{2}}(n)$ such that $\widehat{\sigma_{1}} \circ \widehat{\sigma_{2}}=\omega$. In this equation, $\widehat{\sigma_{2}}$ determines $\widehat{\sigma_{1}}$. But the condition $\omega_{\widetilde{d}_{2}}={\widetilde{\sigma_{1}}}_{1} \circ \widetilde{\sigma}_{2}$ gives the values of $\widehat{\sigma_{2}}$ on $\widetilde{d_{2}}\left(\widehat{\sigma_{2}}(x)=\sigma_{2}(x)\right.$ if $x \in d_{2}$ and $\widehat{\sigma_{2}}(x)=\sigma_{1}^{-1}(\omega(x))$ if $\left.x \in \widetilde{d}_{2} \backslash d_{2}\right)$. Thus, the number of ways to choose $\widehat{\sigma_{2}}$ is the number of ways to extend trivially $\widetilde{\sigma}_{2}$ to a permutation of $2 n$, which is $2^{n-(b+c-e)}(n-(b+c-e))$ ! by Lemma 3.1. This proves equation (5).

Now we have:

$$
\begin{aligned}
& \psi_{n}\left(\alpha_{1}\right) \psi_{n}\left(\alpha_{2}\right)=\frac{1}{2^{2 n-b-c}(n-c) !(n-b) !} \sum_{\widehat{\alpha_{1}} \in \mathcal{S}_{2 n} \cap P_{\alpha_{1}}(n)} \sum_{\widehat{\alpha_{2}} \in \mathcal{S}_{2 n} \cap P_{\alpha_{2}}(n)} \widehat{\sigma_{1}}{\widehat{\sigma_{2}}}
\end{aligned}
$$

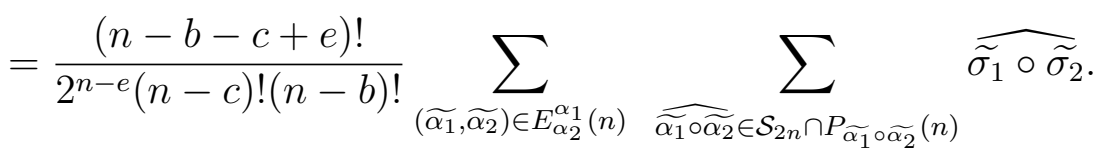

On the other hand:

$$
\psi_{n}\left(\alpha_{1} * \alpha_{2}\right)=\frac{1}{2^{b+c-2 e}(n-c)_{(b-e)}(n-b)_{(c-e)}} \sum_{\left(\widetilde{\alpha_{1}}, \widetilde{\alpha_{2}}\right) \in E_{\alpha_{2}}^{\alpha_{1}}(n)} \psi_{n}\left(\left(\widetilde{\sigma_{1}} \circ \widetilde{\sigma_{2}}, \widetilde{d}_{2}, \widetilde{d}_{1}^{\prime}\right)\right) .
$$

But

$$
\psi_{n}\left(\left(\widetilde{\sigma_{1}} \circ \widetilde{\sigma_{2}}, \widetilde{d_{2}}, \widetilde{d_{1}^{\prime}}\right)\right)=\frac{1}{2^{n-(b+c-e)}(n-(b+c-e)) !} \underset{\widetilde{\widetilde{\alpha_{1}} \odot \widetilde{\alpha_{2}}} \in \mathcal{S}_{2 n} \cap P_{\widetilde{\alpha_{1}} \circ \widetilde{\alpha_{2}}}(n)}{ } \widetilde{\widetilde{\sigma}_{1} \circ \widetilde{\sigma_{2}}}
$$


Thus,

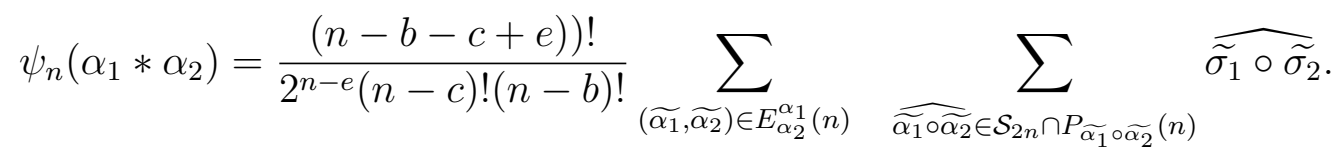

Comparing equations (6) and (7), we see that for any two partial bijections $\alpha_{1}$ and $\alpha_{2}$ of $[2 n]$, we have $\psi_{n}\left(\alpha_{1} * \alpha_{2}\right)=\psi_{n}\left(\alpha_{1}\right) \psi_{n}\left(\alpha_{2}\right)$. In other words, $\psi_{n}$ is a homomorphism of algebras.

\subsection{Proof of the associativity of $*$}

Let $\alpha_{1}, \alpha_{2}$ and $\alpha_{3}$ be three elements of $Q_{n}$. By definition of the product we have:

$$
\begin{aligned}
& \left(\alpha_{1} * \alpha_{2}\right) * \alpha_{3}=\frac{1}{\left|E_{\alpha_{1}}^{\alpha_{2}}(n)\right|} \sum_{\left(\widetilde{\alpha_{1}}, \widetilde{\alpha_{2}}\right) \in E_{\alpha_{1}}^{\alpha_{2}}(n)} \frac{1}{\left|E_{\widetilde{\alpha_{1}} \odot \widetilde{\alpha_{2}}}^{\alpha_{3}}(n)\right|} \sum_{\left(\widetilde{\alpha_{1} \circ \widetilde{\alpha_{2}}}, \widetilde{\alpha_{3}}\right) \in E_{\widetilde{\alpha_{1}} \circ \widetilde{\alpha_{2}}}^{\alpha_{3}}}\left(\widetilde{\widetilde{\sigma_{1}} \circ \widetilde{\sigma_{2}} \circ \widetilde{\sigma_{3}}}, \widetilde{d_{3}}, \widetilde{d_{1}^{\prime}}\right), \\
& \alpha_{1} *\left(\alpha_{2} * \alpha_{3}\right)=\frac{1}{\left|E_{\alpha_{2}}^{\alpha_{3}}(n)\right|} \sum_{\left(\widetilde{\alpha_{2}}, \widetilde{\alpha_{3}}\right) \in E_{\alpha_{2}}^{\alpha_{3}}(n)} \frac{1}{\left|E_{\alpha_{1}}^{\widetilde{\alpha_{2}} \circ \widetilde{\alpha_{3}}}(n)\right|} \sum_{\left(\widetilde{\alpha_{1}}, \widetilde{\alpha_{2} \circ \widetilde{\alpha_{3}}}\right) \in E_{\alpha_{1}}^{\widetilde{\alpha_{2}} \circ \widetilde{\alpha_{3}}}(n)}\left(\widetilde{\sigma_{1}} \circ \widetilde{\widetilde{\sigma_{2}} \circ \widetilde{\sigma_{3}}}, \widetilde{\tilde{d}_{3}}, \widetilde{d_{1}^{\prime}}\right) .
\end{aligned}
$$

We consider now the following sets, indexing the double sums in the equations above:

$$
\begin{array}{r}
X_{1}=\left\{\left(\widetilde{\alpha_{1}}, \widetilde{\alpha_{2}}\right),\left(\widetilde{\widetilde{\alpha_{1}} \circ \widetilde{\alpha_{2}}}, \widetilde{\alpha_{3}}\right)\right) \text { such that }\left(\widetilde{\alpha_{1}}, \widetilde{\alpha_{2}}\right) \in E_{\alpha_{1}}^{\alpha_{2}}(n) \\
\text { and } \left.\left(\widetilde{\alpha_{1} \circ \widetilde{\alpha_{2}}}, \widetilde{\alpha_{3}}\right) \in E_{\widetilde{\alpha_{1}} \circ \widetilde{\alpha_{2}}}^{\alpha_{3}}(n)\right\},
\end{array}
$$

and

$$
\begin{array}{r}
X_{2}=\left\{\left(\widetilde{\alpha_{2}}, \widetilde{\alpha_{3}}\right),\left(\widetilde{\alpha_{1}}, \widetilde{\widetilde{\alpha_{2}} \circ \widetilde{\alpha_{3}}}\right)\right) \text { such that }\left(\widetilde{\alpha_{2}}, \widetilde{\alpha_{3}}\right) \in E_{\alpha_{2}}^{\alpha_{3}}(n) \\
\text { and } \left.\left(\widetilde{\alpha_{1}}, \widetilde{\widetilde{\alpha_{2}} \circ \widetilde{\alpha_{3}}}\right) \in E_{\alpha_{1}}^{\widetilde{\alpha_{2}} \widetilde{\alpha_{3}}}(n)\right\} .
\end{array}
$$

With the notation $X_{1}$, the product $\left(\alpha_{1} * \alpha_{2}\right) * \alpha_{3}$ can be written as follows:

$$
\left(\alpha_{1} * \alpha_{2}\right) * \alpha_{3}=\sum_{\left.\left(\widetilde{\alpha_{1}}, \widetilde{\alpha_{2}}\right),\left(\widetilde{\widetilde{\alpha_{1} \circ \widetilde{\alpha_{2}}}, \widetilde{\alpha_{3}}}\right)\right) \in X_{1}} \frac{1}{\left|E_{\alpha_{1}}^{\alpha_{2}}(n)\right| \cdot\left|E_{\widetilde{\alpha_{1}} \odot \widetilde{\alpha_{2}}}^{\alpha_{3}}(n)\right|}\left(\widetilde{\widetilde{\sigma_{1}} \circ \widetilde{\sigma_{2}}} \circ \widetilde{\sigma_{3}}, \widetilde{d_{3}}, \widetilde{\bar{d}_{1}^{\prime}}\right) .
$$

Schematically, the elements in $X_{1}$ are represented on Figure 5.

In the same way, with the notation $X_{2}$, the product $\alpha_{1} *\left(\alpha_{2} * \alpha_{3}\right)$ can be written in the following way:

$$
\alpha_{1} *\left(\alpha_{2} * \alpha_{3}\right)=\sum_{\left(\widetilde{\left(\widetilde{\alpha_{2}}, \widetilde{\alpha_{3}}\right),\left(\widetilde{\alpha_{1}}, \widetilde{\alpha_{2}} \circ \widetilde{\alpha_{3}}\right)}\right) \in X_{2}} \frac{1}{\left|E_{\alpha_{2}}^{\alpha_{3}}(n)\right| \cdot\left|\overrightarrow{E_{\alpha_{1}}^{\widetilde{\alpha_{2}}} \odot \widetilde{\alpha_{3}}}(n)\right|}\left(\widetilde{\sigma_{1}} \circ \widetilde{\widetilde{\sigma_{2}} \circ \widetilde{\sigma_{3}}}, \widetilde{\tilde{d}_{3}}, \widetilde{d_{1}^{\prime}}\right) .
$$

Schematically, the elements in $X_{2}$ are represented on Figure 6 . 


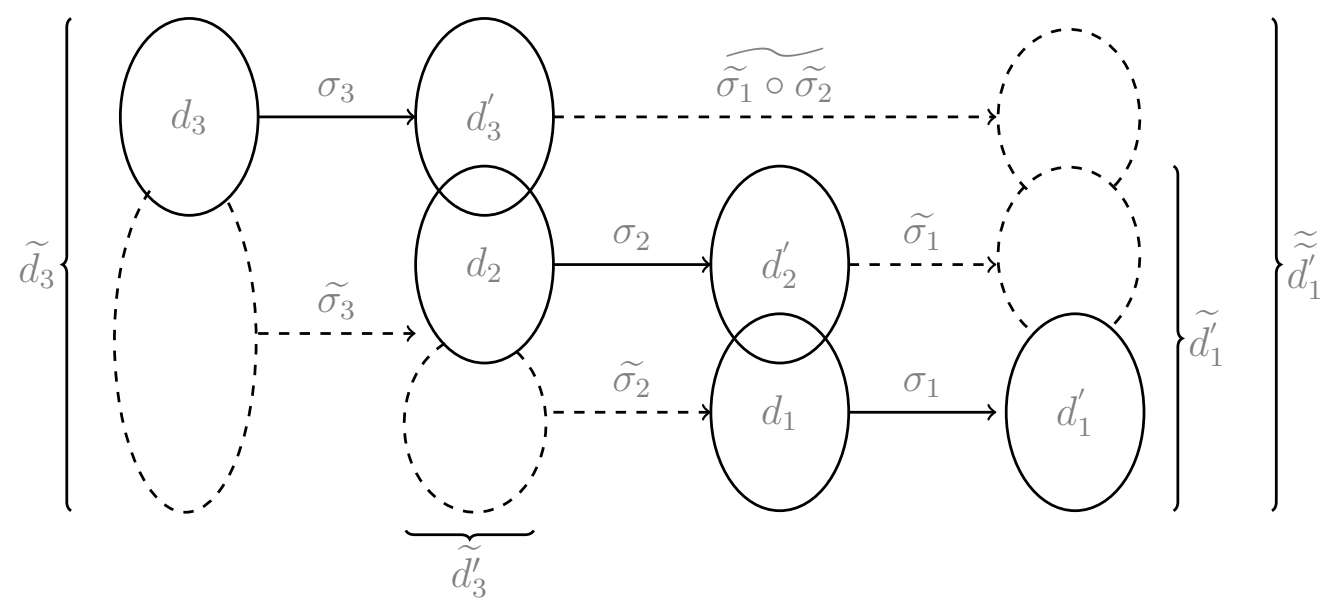

Figure 5: Schematic representation of elements in $X_{1}$.

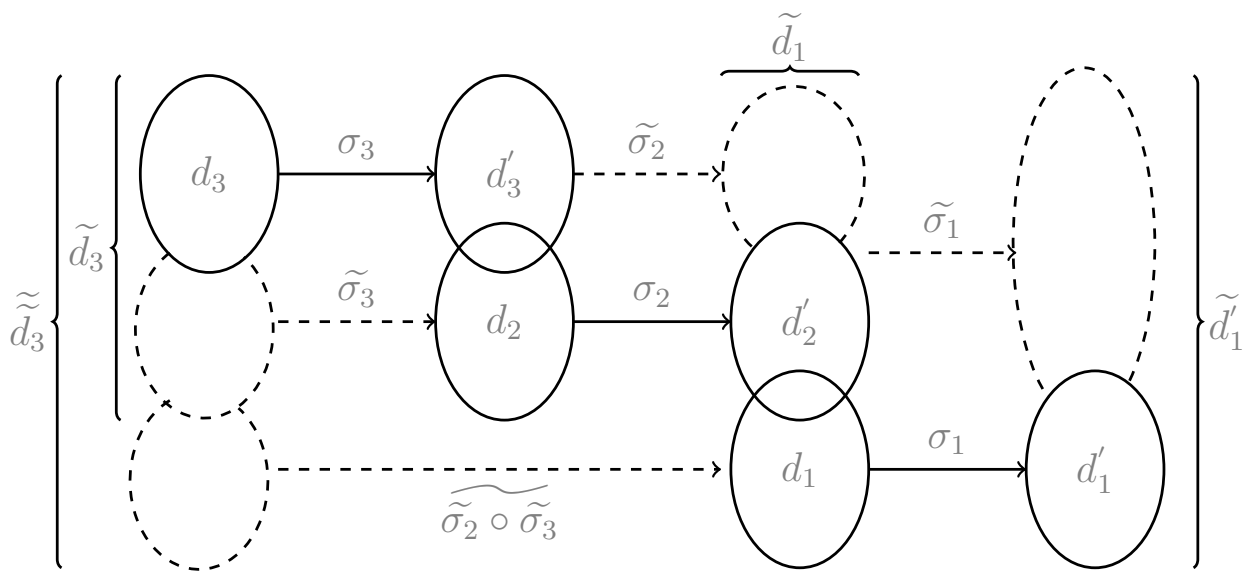

Figure 6: Schematic representation of elements of $X_{2}$.

To prove the associativity of the product, we build a set $X$ and two surjective functions $\phi_{1}: X \longrightarrow X_{1}$ and $\phi_{2}: X \longrightarrow X_{2}$ in order to write both sums (equations (8) and (9)) as sums over the same set $X$. Let $X$ be the set of elements

$$
\left(\epsilon_{1}=\left(\tau_{1}, \delta_{0}, \delta_{1}\right), \epsilon_{2}=\left(\tau_{2}, \delta_{1}, \delta_{2}\right), \epsilon_{3}=\left(\tau_{3}, \delta_{2}, \delta_{3}\right)\right) \in P_{\alpha_{1}}(n) \times P_{\alpha_{2}}(n) \times P_{\alpha_{3}}(n),
$$

satisfying the following properties:

i) $d_{3}^{\prime} \cup d_{2} \subseteq \delta_{2}$.

ii) $d_{2}^{\prime} \cup d_{1} \subseteq \delta_{1}$.

iii) $\delta_{2}=d_{3}^{\prime} \cup \tau_{2}^{-1}\left(d_{1} \cup d_{2}^{\prime}\right)$. 
The elements of this set are schematically represented on Figure 7. Note that (iii)) is a minimality condition. We will see in the proof of Lemma 3.4 below why it is useful.

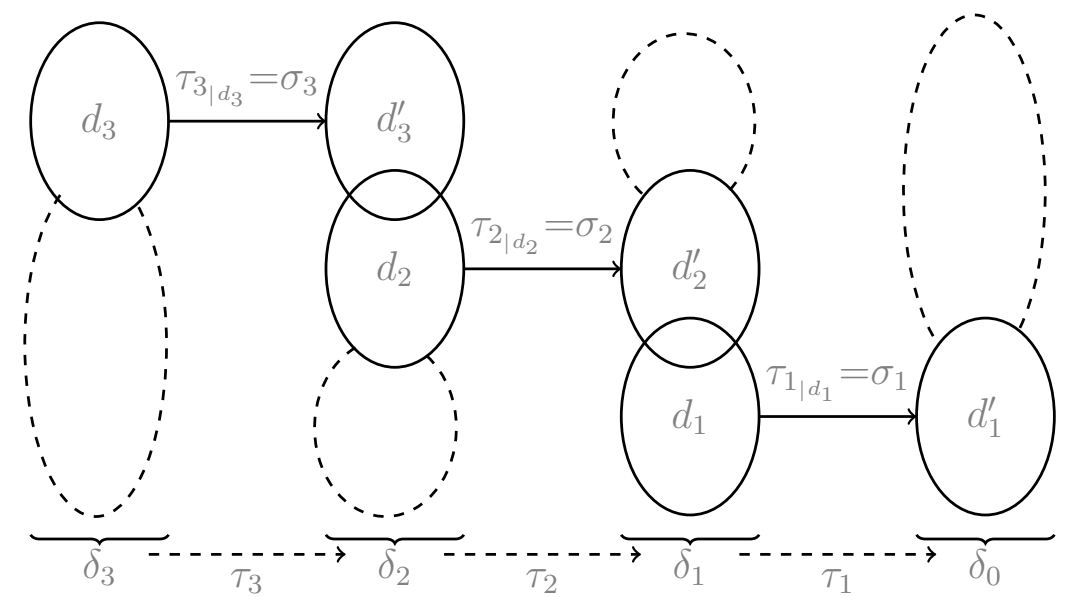

Figure 7: Schematic representation of elements of $X$.

We define the maps $\phi_{1}: X \longrightarrow X_{1}$ and $\phi_{2}: X \longrightarrow X_{2}$ as follows:

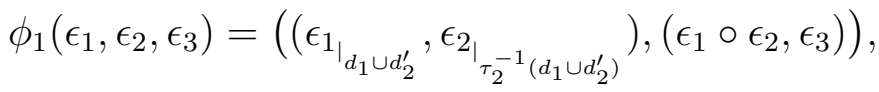

$$
\begin{aligned}
& \phi_{2}\left(\epsilon_{1}, \epsilon_{2}, \epsilon_{3}\right)=\left(\left(\epsilon_{\left.\right|_{d_{2} \cup d_{3}^{\prime}}}, \epsilon_{3_{\tau_{3}^{-1}\left(d_{2} \cup d_{3}^{\prime}\right)}}\right),\left(\epsilon_{2} \circ \epsilon_{3}, \epsilon_{1}\right)\right) .
\end{aligned}
$$

Informally, the map $\phi_{1}$ (resp. $\phi_{2}$ ) forgets the dashed ellipse shape at the top (resp. bottom) of the third (resp. second) column of Figure 7 . We denote by $2 a=\left|d_{3}\right|=$ $\left|d_{3}^{\prime}\right|, 2 b=\left|d_{2}\right|=\left|d_{2}^{\prime}\right|, 2 c=\left|d_{1}\right|=\left|d_{1}^{\prime}\right|, 2 d=\left|d_{3}^{\prime} \cap d_{2}\right|$ and $2 e=\left|d_{2}^{\prime} \cap d_{1}\right|$. We prove the following lemma:

Lemma 3.4. The map $\phi_{1}$ is well defined and surjective. For any element

$$
\left(\left(\widetilde{\alpha_{1}}, \widetilde{\alpha_{2}}\right),\left(\widetilde{\widetilde{\alpha_{1}} \circ \widetilde{\alpha_{2}}}, \widetilde{\alpha_{3}}\right)\right) \in X_{1}
$$

we have:

$$
\left.\mid \phi_{1}^{-1}\left(\left(\widetilde{\alpha_{1}}, \widetilde{\alpha_{2}}\right),\left(\widetilde{\widetilde{\alpha_{1}} \circ \widetilde{\alpha_{2}}}, \widetilde{\alpha_{3}}\right)\right)\right) \mid=2^{a-d-f} \cdot(n-b-c+e)_{(a-d-f)},
$$

where $2 f=\left|\left(\widetilde{d}_{2} \backslash d_{2}\right) \cap d_{3}^{\prime}\right|$.

Note that $f$, unlike $a, b, c, d, e$, depends on the element of $X_{1}$ that we consider.

Proof. For any $\left(\epsilon_{1}, \epsilon_{2}, \epsilon_{3}\right) \in X$, we must check that $\phi_{1}\left(\left(\epsilon_{1}, \epsilon_{2}, \epsilon_{3}\right)\right)$ is in $X_{1}$. Set

$$
\left(\widetilde{\alpha_{1}}, \widetilde{\alpha_{2}}\right)=\left(\epsilon_{1_{d_{1} \cup d_{2}^{\prime}}}, \epsilon_{\left.\right|_{\tau_{2}^{-1}\left(d_{1} \cup d_{2}^{\prime}\right)}}\right) .
$$


First $\left(\widetilde{\alpha_{1}}, \widetilde{\alpha_{2}}\right)$ is in $P_{\alpha_{1}}(n) \times P_{\alpha_{2}}(n)$ since $\left(\epsilon_{1}, \epsilon_{2}\right)$ is in $P_{\alpha_{1}}(n) \times P_{\alpha_{2}}(n)$. Then, the codomain of $\widetilde{\alpha_{2}}$ and the domain of $\widetilde{\alpha_{1}}$ are $d_{1} \cup d_{2}^{\prime}$. Thus, $\left(\widetilde{\alpha_{1}}, \widetilde{\alpha_{2}}\right)$ is in $E_{\alpha_{1}}^{\alpha_{2}}(n)$.

Second, it is easy to check that $\left(\epsilon_{1} \circ \epsilon_{2}, \epsilon_{3}\right)$ is in $P_{\widetilde{\alpha_{1}} \circ \widetilde{\alpha_{2}}}(n) \times P_{\alpha_{3}}(n)$. Then, to be in $E_{\widetilde{\alpha_{1}} \circ \widetilde{\alpha_{2}}}^{\alpha_{3}}(n),\left(\epsilon_{1} \circ \epsilon_{2}, \epsilon_{3}\right)$ must verify the condition that $\delta_{2}=d_{3}^{\prime} \cup \tau_{2}^{-1}\left(d_{1} \cup d_{2}^{\prime}\right)$, which is given by condition (iii)).

Thus, $\phi_{1}$ is well defined.

Now, fix $\left(\left(\widetilde{\alpha_{1}}, \widetilde{\alpha_{2}}\right),\left(\widetilde{\widetilde{\alpha_{1}} \circ \widetilde{\alpha_{2}}}, \widetilde{\alpha_{3}}\right)\right) \in X_{1}$. We will count the number of its pre-images by $\phi_{1}$. To construct an element of $\phi_{1}^{-1}\left(\left(\left(\widetilde{\alpha_{1}}, \widetilde{\alpha_{2}}\right),\left(\widetilde{\widetilde{\alpha_{1}} \circ \widetilde{\alpha_{2}}}, \widetilde{\alpha_{3}}\right)\right)\right)$, we have only to build $\epsilon_{1}, \epsilon_{2}$ and $\delta_{1}$ since the other elements $\epsilon_{3}, \delta_{0}, \delta_{2}$ and $\delta_{3}$ are determined by $\widetilde{\alpha_{1}}, \widetilde{\alpha_{2}}, \widetilde{\alpha_{3}}$ and $\widetilde{\alpha_{1} \circ \widetilde{\alpha_{2}}}$. First, to build $\delta_{1}$, we must extend $d_{1} \cup d_{2}^{\prime}$ by adding pairs of form $\rho(k)$ to obtain a set which has the same cardinality as $\delta_{2}=\widetilde{d}_{2} \cup d_{3}^{\prime}$ which is $2 a+2 b+2 c-2 e-2 d-2 f$. We have $\left|d_{1} \cup d_{2}^{\prime}\right|=2 b+2 c-2 e$, so the number of possible ways to extend $d_{1} \cup d_{2}^{\prime}$ is the number of choices of $2 a+2 b+2 c-2 e-2 d-2 f-(2 b+2 c-2 e)=2 a-2 d-2 f$ elements among $2 n-(2 b+2 c-2 e)$. Since our choice must respect the condition that the extended set is in $\mathbf{P}_{n}$, this number is:

$$
\left(\begin{array}{c}
n-(b+c-e) \\
a-d-f
\end{array}\right)
$$

Once the set $d_{1} \cup d_{2}^{\prime}$ is extended, we should extend $\widetilde{\sigma_{1}}$ to $\tau_{1}$ (we have the definition domain $\delta_{0}$ and the arrival domain $\delta_{1}$ ) by sending the pairs of form $\rho(k)$ to pairs with same form. The number of ways to do so is:

$$
2^{a-d-f} \cdot(a-d-f) !
$$

After extending $\widetilde{\sigma_{1}}$ to $\tau_{1}$, we get immediately $\tau_{2}$ because $\tau_{1} \circ \tau_{2}=\widetilde{\widetilde{\sigma_{1}} \circ \widetilde{\sigma_{2}}}$ is given. Thus, the cardinality of the set $\phi_{1}^{-1}\left(\left(\left(\widetilde{\alpha_{1}}, \widetilde{\alpha_{2}}\right),\left(\widetilde{\widetilde{\alpha_{1}} \circ \widetilde{\alpha_{2}}}, \widetilde{\alpha_{3}}\right)\right)\right)$ is equal to:

$$
\left(\begin{array}{c}
n-(b+c-e) \\
a-d-f
\end{array}\right) \cdot 2^{a-d-f} \cdot(a-d-f) !=2^{a-d-f} \cdot(n-b-c+e)_{(a-d-f)} .
$$

In the same way, we can prove the following lemma.

Lemma 3.5. The map $\phi_{2}$ is well defined and surjective. For any element $\left(\left(\widetilde{\alpha_{2}}, \widetilde{\alpha_{3}}\right),\left(\widetilde{\alpha_{1}}, \widetilde{\widetilde{\alpha_{2}} \circ \widetilde{\alpha_{3}}}\right)\right) \in X_{2}$, we have:

$$
\left|\phi_{2}^{-1}\left(\left(\left(\widetilde{\alpha_{2}}, \widetilde{\alpha_{3}}\right),\left(\widetilde{\alpha_{1}}, \widetilde{\widetilde{\alpha_{2}} \circ \widetilde{\alpha_{3}}}\right)\right)\right)\right|=2^{c-e-g} \cdot(n-a-b+d)_{(c-e-g)},
$$

where, $2 g=\left|\left(\widetilde{d_{2}^{\prime}} \backslash d_{2}^{\prime}\right) \cap d_{1}\right|$.

We can also verify using the notations above that:

$$
\left|E_{\alpha_{1}}^{\alpha_{2}}(n)\right|=2^{b+c-2 e} \cdot(n-c)_{(b-e)} \cdot(n-b)_{(c-e)},
$$




$$
\left|E_{\alpha_{2}}^{\alpha_{3}}(n)\right|=2^{a+b-2 d} \cdot(n-b)_{(a-d)} \cdot(n-a)_{(b-d)},
$$

and

$$
\begin{aligned}
& \left|E_{\widetilde{\alpha_{1}} \odot \widetilde{\alpha_{2}}}^{\alpha_{3}}(n)\right|=2^{a+b+c-2 d-e-2 f} \cdot(n-b-c+e)_{(a-d-f)} \cdot(n-a)_{(b+c-d-e-f)}, \\
& \left|E_{\alpha_{1}}^{\widetilde{\alpha_{2}} \odot \widetilde{\alpha_{3}}}(n)\right|=2^{a+b+c-d-2 e-2 g} \cdot(n-a-b+d)_{(c-e-g)} \cdot(n-c)_{(a+b-d-e-g)} .
\end{aligned}
$$

The products $\left(\alpha_{1} * \alpha_{2}\right) * \alpha_{3}$ and $\alpha_{1} *\left(\alpha_{2} * \alpha_{3}\right)$ given in equations (8) and (9) as sums over $X_{1}$ and $X_{2}$ can be written as sums over the set $X$ as follows:

$$
\begin{aligned}
\left(\alpha_{1} * \alpha_{2}\right) * \alpha_{3}=\sum_{\left(\epsilon_{1}, \epsilon_{2}, \epsilon_{3}\right) \in X} & \frac{1}{2^{2 a+2 b+2 c-3 d-3 e-3 f}} \cdot\left((n-b-c+e)_{(a-d-f)}\right)^{2} \\
& \cdot(n-a)_{(b+c-d-e-f)} \cdot(n-c)_{(b-e)} \cdot(n-b)_{(c-e)} \\
& \epsilon_{1} \circ \epsilon_{2} \circ \epsilon_{3},
\end{aligned}
$$

and

$$
\begin{aligned}
\alpha_{1} *\left(\alpha_{2} * \alpha_{3}\right)=\sum_{\left(\epsilon_{1}, \epsilon_{2}, \epsilon_{3}\right) \in X} & \frac{1}{2^{2 a+2 b+2 c-3 d-3 e-3 g}} \cdot\left((n-a-b+d)_{(c-e-g)}\right)^{2} \\
& \cdot(n-c)_{(a+b-d-e-g)} \cdot(n-a)_{(b-d)} \cdot(n-b)_{(a-d)} \\
& \epsilon_{1} \circ \epsilon_{2} \circ \epsilon_{3} .
\end{aligned}
$$

For any positive integer $n$, we have the following easy identities:

$$
\begin{aligned}
(n-b-c+e)_{(a-d-f)} \cdot(n-c)_{(b-e)} & =(n-c)_{(a+b-d-e-f)} \\
(n-a)_{(b-d)} \cdot(n-a-b+d)_{(c-e-f)} & =(n-a)_{(b+c-d-e-f)} \\
(n-b)_{(c-e)} \cdot(n-b-c+e)_{(a-d-f)} & =(n-b)_{(a-d)} \cdot(n-a-b+d)_{(c-e-f)} .
\end{aligned}
$$

Thus, the product $\left(\alpha_{1} * \alpha_{2}\right) * \alpha_{3}$ can be written as follows:

$$
\begin{aligned}
\left(\alpha_{1} * \alpha_{2}\right) * \alpha_{3}=\sum_{\left(\epsilon_{1}, \epsilon_{2}, \epsilon_{3}\right) \in X} & \frac{1}{2^{2 a+2 b+2 c-3 d-3 e-3 f}} \cdot\left((n-a-b+d)_{(c-e-f)}\right)^{2} \\
& \cdot(n-c)_{(a+b-d-e-f)} \cdot(n-a)_{(b-d)} \cdot(n-b)_{(a-d)} \\
& \epsilon_{1} \circ \epsilon_{2} \circ \epsilon_{3} .
\end{aligned}
$$

For any element of $X$, the equality $\left|\delta_{2}\right|=\left|\delta_{1}\right|$ can be written $2 c+2 b-2 e+2 a-2 d-2 f=$ $2 a+2 b-2 d+2 c-2 e-2 g$, so we have $f=g$. Comparing (10) and (11), we see that products $\left(\alpha_{1} * \alpha_{2}\right) * \alpha_{3}$ and $\alpha_{1} *\left(\alpha_{2} * \alpha_{3}\right)$ are equal, therefore we get the associativity.

\subsection{Action of $\mathcal{B}_{n} \times \mathcal{B}_{n}$ on $\mathcal{D}_{n}$}

In this section, we build the algebra $\mathcal{A}_{n}$ as the algebra of invariant elements by an action of $\mathcal{B}_{n} \times \mathcal{B}_{n}$ on $\mathcal{D}_{n}$. 
Definition 3.5. The group $\mathcal{B}_{n} \times \mathcal{B}_{n}$ acts on $Q_{n}$ by:

$$
(a, b) \bullet\left(\sigma, d, d^{\prime}\right)=\left(a \sigma b^{-1}, b(d), a\left(d^{\prime}\right)\right),
$$

for any $(a, b) \in \mathcal{B}_{n} \times \mathcal{B}_{n}$ and $\left(\sigma, d, d^{\prime}\right) \in Q_{n}$.

Observation 3.5. Two partial bijections are in the same orbit if and only if they have the same coset-type.

We extend this action by linearity to get an action of $\mathcal{B}_{n} \times \mathcal{B}_{n}$ on $\mathcal{D}_{n}$.

Lemma 3.6. For any three permutations $a, b$ and $c$ of $\mathcal{B}_{n}$ and for any partial bijections $\alpha_{1}, \alpha_{2}$ of $[2 n]$, the set $E_{\alpha_{1}}^{\alpha_{2}}(n)$ is in bijection with $E_{(a, b) \bullet \alpha_{1}}^{(b, c) \bullet \alpha_{2}}(n)$.

Proof. We check easily that the two following functions:

$$
\Theta: \begin{array}{ccc}
E_{\alpha_{1}}^{\alpha_{2}}(n) & \rightarrow & E_{(a, b) \bullet \alpha_{1}}^{(b, c) \bullet \alpha_{2}}(n) \\
\left(\widetilde{\alpha_{1}}, \widetilde{\alpha_{2}}\right) & \mapsto \quad\left((a, b) \bullet \widetilde{\alpha_{1}},(b, c) \bullet \widetilde{\alpha_{2}}\right)
\end{array}
$$

and

$$
\Psi: \begin{array}{ccc}
E_{(a, b) \bullet \alpha_{1}}^{(b, c) \bullet \alpha_{2}}(n) & \rightarrow & E_{\alpha_{1}}^{\alpha_{2}}(n) \\
\left(\beta_{1}, \beta_{2}\right) & \mapsto \quad\left(\left(a^{-1}, b^{-1}\right) \bullet \beta_{1},\left(b^{-1}, c^{-1}\right) \bullet \beta_{2}\right)
\end{array},
$$

are well defined. Moreover, they are inverse from each other:

$$
\begin{aligned}
\Psi\left(\Theta\left(\left(\widetilde{\alpha_{1}}, \widetilde{\alpha_{2}}\right)\right)\right) & =\Psi\left(\left((a, b) \bullet \widetilde{\alpha_{1}},(b, c) \bullet \widetilde{\alpha_{2}}\right)\right) \\
& =\left(\left(a^{-1}, b^{-1}\right) \bullet(a, b) \bullet \widetilde{\alpha_{1}},\left(b^{-1}, c^{-1}\right) \bullet(b, c) \bullet \widetilde{\alpha_{2}}\right) \\
& =\left(\widetilde{\alpha_{1}}, \widetilde{\alpha_{2}}\right),
\end{aligned}
$$

and, similarly,

$$
\Theta\left(\Psi\left(\beta_{1}, \beta_{2}\right)\right)=\left(\beta_{1}, \beta_{2}\right)
$$

Thus $\Theta$ defines a bijection between $E_{\alpha_{1}}^{\alpha_{2}}(n)$ and $E_{(a, b) \bullet \alpha_{1}}^{(b, c) \bullet \alpha_{2}}(n)$ with inverse $\Psi$.

It follows from this lemma that the action $\bullet$ is compatible with the product of $Q_{n}$. Namely, we can prove the following corollary.

Corollary 3.7. For any $(a, b, c) \in \mathcal{B}_{n}^{3}$ and for any partial bijections $\alpha_{1}, \alpha_{2}$ of $[2 n]$, we have:

$$
(a, c) \bullet\left(\alpha_{1} * \alpha_{2}\right)=\left((a, b) \bullet \alpha_{1}\right) *\left((b, c) \bullet \alpha_{2}\right) .
$$

Proof. If $\left(\widetilde{\alpha_{1}}, \widetilde{\alpha_{2}}\right) \in E_{\alpha_{1}}^{\alpha_{2}}(n)$, we have:

$$
\begin{aligned}
\left((a, b) \bullet \widetilde{\alpha_{1}}\right) \circ\left((b, c) \bullet \widetilde{\alpha_{2}}\right) & =\left(a \widetilde{\sigma_{1}} b^{-1}, b\left(\widetilde{d_{1}}\right), a\left(\widetilde{d_{1}^{\prime}}\right)\right) \circ\left(b \widetilde{\sigma_{2}} c^{-1}, c\left(\widetilde{d_{2}}\right), b\left(\widetilde{d_{2}^{\prime}}\right)\right) \\
& =\left(a \widetilde{\sigma_{1}} \widetilde{\sigma_{2}} c^{-1}, c\left(\widetilde{d_{2}}\right), a\left(\widetilde{d_{1}^{\prime}}\right)\right) \\
& =(a, c) \bullet\left(\widetilde{\alpha_{1}} \circ \widetilde{\alpha_{2}}\right) .
\end{aligned}
$$


Then, we can write:

$$
\begin{aligned}
& (a, c) \bullet\left(\alpha_{1} * \alpha_{2}\right)=\frac{1}{\left|E_{\alpha_{1}}^{\alpha_{2}}(n)\right|} \sum_{\left(\widetilde{\alpha_{1}}, \widetilde{\alpha_{2}}\right) \in E_{\alpha_{1}}^{\alpha_{2}}(n)}(a, c) \bullet\left(\widetilde{\alpha_{1}} \circ \widetilde{\alpha_{2}}\right) \\
& =\frac{1}{\left|E_{\alpha_{1}}^{\alpha_{2}}(n)\right|} \sum_{\left(\widetilde{\alpha_{1}}, \widetilde{\alpha_{2}}\right) \in E_{\alpha_{1}}^{\alpha_{2}}(n)}\left((a, b) \bullet \widetilde{\alpha_{1}}\right) \circ\left((b, c) \bullet \widetilde{\alpha_{2}}\right)
\end{aligned}
$$

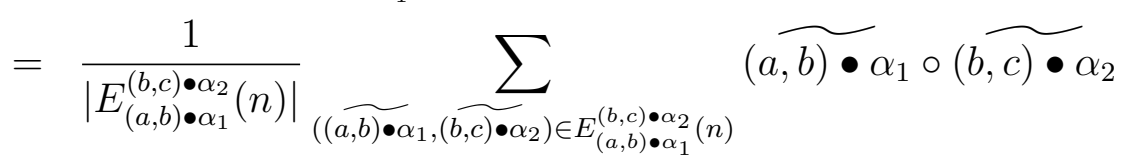

$$
\begin{aligned}
& \left.=\left((a, b) \bullet \alpha_{1}\right) *\left((b, c) \bullet \alpha_{2}\right)\right) \text {. }
\end{aligned}
$$

We consider the set $\mathcal{A}_{n}$ of invariant elements by the action of $\mathcal{B}_{n} \times \mathcal{B}_{n}$ on $\mathcal{D}_{n}$ :

$$
\mathcal{A}_{n}=\mathcal{D}_{n}^{\mathcal{B}_{n} \times \mathcal{B}_{n}}=\left\{x \in \mathcal{D}_{n} \mid(a, b) \bullet x=x \text { for any }(a, b) \in \mathcal{B}_{n} \times \mathcal{B}_{n}\right\} .
$$

For every partition $\lambda$ such that $|\lambda| \leqslant n$, we define the set $A_{\lambda, n}$ to be the set of all partial bijections $\alpha$ of $[2 n]$ such that $\operatorname{ct}(\alpha)=\lambda$. The sum of all elements in $A_{\lambda, n}$ is denoted by $S_{\lambda, n}$.

Proposition 3.8. The set $\mathcal{A}_{n}$ is an algebra with basis the elements $\left(S_{\lambda, n}\right)_{|\lambda| \leqslant n}$.

Proof. For every $(a, b) \in \mathcal{B}_{n} \times \mathcal{B}_{n}$, and for every $x, y \in \mathcal{A}_{n}$, we have by linearity:

$$
(a, b) \bullet(x * y)=((a, i d) \bullet x) *((i d, b) \bullet y)=x * y .
$$

So $\mathcal{A}_{n}$ is an algebra.

Any element $x \in \mathcal{D}_{n}$ writes $x=\sum_{k=1}^{n} \sum_{\substack{d, d^{\prime} \in \mathbf{P}_{n} \\|d|=\left|d^{\prime}\right|=2 k}} \sum_{\substack{\sigma: d \rightarrow d^{\prime} \\ \text { bijection }}} c_{\left(\sigma, d, d^{\prime}\right)}\left(\sigma, d, d^{\prime}\right)$. If, furthermore $x$ is in $\mathcal{A}_{n}$, then for every $(a, b) \in \mathcal{B}_{n} \times \mathcal{B}_{n}$ we have:

$$
\sum_{k=1}^{n} \sum_{\substack{d, d^{\prime} \in \mathbf{P}_{n} \\|d|=\left|d^{\prime}\right|=2 k}} \sum_{\substack{\sigma: d \rightarrow d^{\prime} \\ \text { bijection }}} c_{\left(\sigma, d, d^{\prime}\right)}\left(a \sigma b^{-1}, b(d), a\left(d^{\prime}\right)\right)=\sum_{k=1}^{n} \sum_{\substack{d, d^{\prime} \in \mathbf{P}_{n} \\|d|=\left|d^{\prime}\right|=2 k}} \sum_{\substack{\sigma: d \rightarrow d^{\prime} \\ \text { bijection }}} c_{\left(\sigma, d, d^{\prime}\right)}\left(\sigma, d, d^{\prime}\right) .
$$

Thus, for any $(a, b) \in \mathcal{B}_{n} \times \mathcal{B}_{n}$, we have $c_{\left(a \sigma b^{-1}, b(d), a\left(d^{\prime}\right)\right)}=c_{\left(\sigma, d, d^{\prime}\right)}$. This means that if $x \in \mathcal{A}_{n}$, all partial permutations in the same orbit - that is with the same coset-type have the same coefficients. Therefore, the elements $\left(S_{\lambda, n}\right)_{|\lambda| \leqslant n}$ form a basis of $\mathcal{A}_{n}$.

Corollary 3.9. If $\lambda$ and $\delta$ are two partitions such that $|\lambda|,|\delta| \leqslant n$, there exist unique constants $c_{\lambda \delta}^{\rho}(n) \in \mathbb{C}$ such that:

$$
S_{\lambda, n} * S_{\delta, n}=\sum_{\substack{\rho \text { partition } \\ \max (|\lambda|,|\delta|) \leqslant|\rho| \leqslant \min (|\lambda|+|\delta|, n)}} c_{\lambda \delta}^{\rho}(n) S_{\rho, n} .
$$


Proof. We only have to prove the inequalities on the size of $\rho$. Let $\alpha_{1}$ and $\alpha_{2}$ be two partial bijections of $[2 n]$ with coset-type $\lambda$ and $\delta$. By definition (see Figure 4), every partial bijection of $[2 n]$ that appears in the sum of the product $\alpha_{1} * \alpha_{2}$ has some cosettype $\rho$ with $|\rho|=\frac{\left|d_{1} \cup d_{2}^{\prime}\right|}{2}$. But

$$
\max \left(\frac{\left|d_{1}\right|}{2}, \frac{\left|d_{2}^{\prime}\right|}{2}\right)=\max (|\lambda|,|\delta|) \leqslant|\rho|=\frac{\left|d_{1} \cup d_{2}^{\prime}\right|}{2} \leqslant \frac{\left|d_{1}\right|+\left|d_{2}^{\prime}\right|}{2}=|\lambda|+|\delta| .
$$

Lemma 3.10. Let $\lambda$ be a partition such that $|\lambda|=r \leqslant n$, we have:

$$
\psi_{n}\left(S_{\lambda, n}\right)=\frac{1}{2^{n-|\lambda|}(n-|\lambda|) !}\left(\begin{array}{c}
n-|\bar{\lambda}| \\
m_{1}(\lambda)
\end{array}\right) K_{\bar{\lambda}}(n) .
$$

Proof. We first prove the following equation:

$$
\sum_{\alpha \in A_{\lambda, n}} \sum_{\hat{\alpha} \in \mathcal{S}_{2 n} \cap P_{\alpha}(n)} \hat{\sigma}=\left(\begin{array}{c}
n-|\bar{\lambda}| \\
m_{1}(\lambda)
\end{array}\right) K_{\bar{\lambda}}(n) .
$$

Fix a permutation $\omega \in K_{\bar{\lambda}}(n)$, that is $\omega \in \mathcal{S}_{2 n}$ and $\operatorname{ct}(\omega)=\bar{\lambda} \cup 1^{n-|\bar{\lambda}|}$. We are looking for the number of partial bijections $\alpha \in A_{\lambda, n}$ such that $\omega$ is one of its trivial extensions. There is a unique set $S$ such that $\operatorname{ct}\left(\omega_{\left.\right|_{S}}\right)=\bar{\lambda}$. We call this set the support of $\omega$ and denote it $\operatorname{supp}(\omega)$. The following condition is necessary so that $\omega$ is a trivial extension of $\alpha: \operatorname{supp}(\omega) \subseteq d$ and $\alpha_{\left.\right|_{\operatorname{supp}(\omega)}}$ must be equal to $\omega_{\mid \operatorname{supp}(\omega)}$. Thus the partial bijections $\alpha$ we are looking for are the restrictions of $\omega$ to sets $\operatorname{supp}(\omega) \sqcup x$, with $|\operatorname{supp}(\omega) \sqcup x|=2|\lambda|$. Since $|\operatorname{supp}(\omega)|=2|\bar{\lambda}|$, one has the necessarily $|x|=2(|\lambda|-|\bar{\lambda}|)$. So the number of such $\alpha$ is $\left(\begin{array}{c}n-|\bar{\lambda}| \\ |\lambda|-|\bar{\lambda}|\end{array}\right)=\left(\begin{array}{c}n-|\bar{\lambda}| \\ m_{1}(\lambda)\end{array}\right)$. This ends the proof of equation (12).

By applying $\psi_{n}$ to $S_{\lambda, n}$, we get:

$$
\begin{aligned}
\psi_{n}\left(S_{\lambda, n}\right) & =\psi_{n}\left(\sum_{\alpha \in A_{\lambda, n}} \alpha\right) \\
& =\frac{1}{2^{n-|\lambda|}(n-|\lambda|) !} \sum_{\alpha \in A_{\lambda, n}} \sum_{\hat{\alpha} \in \mathcal{S}_{2 n} \cap P_{\alpha}(n)} \hat{\sigma} \\
& =\frac{1}{2^{n-|\lambda|}(n-|\lambda|) !}\left(\begin{array}{c}
n-|\bar{\lambda}| \\
m_{1}(\lambda)
\end{array}\right) K_{\bar{\lambda}}(n) .
\end{aligned}
$$

Lemma 3.10 implies that $\psi_{n}\left(\mathcal{A}_{n}\right) \subseteq \mathbb{C}\left[\mathcal{B}_{n} / \mathcal{S}_{2 n} \backslash \mathcal{B}_{n}\right]$. The homomorphism $\mathcal{A}_{n} \rightarrow$ $\mathbb{C}\left[\mathcal{B}_{n} / \mathcal{S}_{2 n} \backslash \mathcal{B}_{n}\right]$ mentioned in Section 2.5 is the restriction $\psi_{n_{\left.\right|_{\mathcal{A}_{n}}}}$, which is surjective but not injective. 


\subsection{Homomorphism from $\mathcal{A}_{n+1}$ to $\mathcal{A}_{n}$}

This subsection is dedicated to the proof of the following proposition:

Proposition 3.11. The function $\varphi_{n}$ defined as follows:

$$
\begin{aligned}
& \varphi_{n}: \mathcal{A}_{n+1} \rightarrow \\
& S_{\lambda, n+1} \mapsto\left\{\begin{array}{lll}
\frac{n+1}{(n+1-|\lambda|)} S_{\lambda, n} & \text { if } & |\lambda|<n+1, \\
0 & \text { if } & |\lambda|=n+1,
\end{array}\right.
\end{aligned}
$$

is a homomorphism of algebras.

Let $S_{\lambda, n+1}$ and $S_{\delta, n+1}$ where $|\lambda| \leqslant n+1$ and $|\delta| \leqslant n+1$ be two basis elements of $\mathcal{A}_{n+1}$.

If $\lambda$ (resp. $\delta$ ) is a partition of $n+1$, then $\varphi_{n}\left(S_{\lambda, n+1}\right)\left(\operatorname{resp} . \varphi_{n}\left(S_{\delta, n+1}\right)\right)$ is equal to zero, and by Corollary 3.9 we have:

$$
S_{\lambda, n+1} * S_{\delta, n+1}=\sum_{\substack{\rho \text { partition } \\|\rho|=n+1}} c_{\lambda \delta}^{\rho}(n+1) S_{\rho, n+1} .
$$

Note that the size of all partitions $\rho$ in the sum index of this equation is $n+1$. By applying $\varphi_{n}$, we get:

$$
\varphi_{n}\left(S_{\lambda, n+1} * S_{\delta, n+1}\right)=\sum_{\substack{\rho \text { partition } \\|\rho|=n+1}} c_{\lambda \delta}^{\rho}(n+1) \varphi_{n}\left(S_{\rho, n+1}\right)=0 .
$$

Thus in this case we have $\varphi_{n}\left(S_{\lambda, n+1} * S_{\delta, n+1}\right)=\varphi_{n}\left(S_{\lambda, n+1}\right) * \varphi_{n}\left(S_{\delta, n+1}\right)$.

In the other case $(|\lambda| \leqslant n$ and $|\delta| \leqslant n)$ we have by Corollary 3.9:

$$
S_{\lambda, n+1} * S_{\delta, n+1}=\sum_{\substack{r \leqslant n+1 \\ \rho \vdash r}} c_{\lambda \delta}^{\rho}(n+1) S_{\rho, n+1} .
$$

This gives us the following equation after applying $\varphi_{n}$ :

$$
\begin{aligned}
\varphi_{n}\left(S_{\lambda, n+1} * S_{\delta, n+1}\right) & =\varphi_{n}\left(\sum_{\substack{r \leqslant n+1 \\
\rho \vdash r}} c_{\lambda \delta}^{\rho}(n+1) S_{\rho, n+1}\right) \\
& =\sum_{\substack{r \leqslant n \\
\rho \vdash r}} c_{\lambda \delta}^{\rho}(n+1) \frac{n+1}{(n+1-|\rho|)} S_{\rho, n} .
\end{aligned}
$$

In the other hand, we have:

$$
\begin{aligned}
\varphi_{n}\left(S_{\lambda, n+1}\right) * \varphi_{n}\left(S_{\delta, n+1}\right) & =\frac{n+1}{(n+1-|\lambda|)} S_{\lambda, n} * \frac{n+1}{(n+1-|\delta|)} S_{\delta, n} \\
& =\frac{n+1}{(n+1-|\lambda|)} \frac{n+1}{(n+1-|\delta|)} \sum_{\substack{r \leqslant n \\
\rho \vdash r}} c_{\lambda \delta}^{\rho}(n) S_{\rho, n} .
\end{aligned}
$$


Thus, $\varphi_{n}$ is a homomorphism if we have the following equality for any partition $\rho$ with size at most $n$ :

$$
\frac{c_{\lambda \delta}^{\rho}(n+1)}{c_{\lambda \delta}^{\rho}(n)}=\frac{\frac{n+1}{(n+1-|\lambda|)} \frac{n+1}{(n+1-|\delta|)}}{\frac{n+1}{(n+1-|\rho|)}} .
$$

Let $\rho$ be a partition with size at most $n$ and $\alpha$ an element of $A_{\rho, n}$. We define $H_{\lambda \delta}^{\rho}(n)$ to be the following set:

$\left\{\left(\alpha_{1}, \alpha_{2}\right) \in A_{\lambda, n} \times A_{\delta, n}\right.$ such that there exists $\left(\widetilde{\alpha_{1}}, \widetilde{\alpha_{2}}\right) \in E_{\alpha_{1}}^{\alpha_{2}}(n)$ with $\left.\alpha=\widetilde{\alpha_{1}} \circ \widetilde{\alpha_{2}}\right\}$.

This set depends on $\alpha$ by definition. However, $\alpha$ does not appear in our notation. This should not be an issue, since $\alpha$ is fixed in the whole proof.

The coefficient $c_{\lambda \delta}^{\rho}(n)$ can be written as follows:

$$
c_{\lambda \delta}^{\rho}(n)=\sum_{\left(\alpha_{1}, \alpha_{2}\right) \in H_{\lambda \delta}^{\rho}(n)} \frac{1}{\left|E_{\alpha_{1}}^{\alpha_{2}}(n)\right|} .
$$

Similarly, we have:

$$
c_{\lambda \delta}^{\rho}(n+1)=\sum_{\left(\alpha_{1}, \alpha_{2}\right) \in H_{\lambda \delta}^{\rho}(n+1)} \frac{1}{\left|E_{\alpha_{1}}^{\alpha_{2}}(n+1)\right|} .
$$

By equation (4), if $\left(\alpha_{1}, \alpha_{2}\right) \in H_{\lambda \delta}^{\rho}(n)$, we have:

$$
\left|E_{\alpha_{1}}^{\alpha_{2}}(n)\right|=2^{2|\rho|-|\lambda|-|\delta|}(n-|\lambda|)_{(|\rho|-|\lambda|)}(n-|\delta|)_{(|\rho|-|\delta|)} .
$$

Similarly, if $\left(\alpha_{1}, \alpha_{2}\right) \in H_{\lambda \delta}^{\rho}(n+1)$, we have:

$$
\left|E_{\alpha_{1}}^{\alpha_{2}}(n+1)\right|=2^{2|\rho|-|\lambda|-|\delta|}(n+1-|\lambda|)_{(|\rho|-|\lambda|)}(n+1-|\delta|)_{(|\rho|-|\delta|)} .
$$

Thus, we get:

$$
c_{\lambda \delta}^{\rho}(n)=\frac{\left|H_{\lambda \delta}^{\rho}(n)\right|}{2^{2|\rho|-|\lambda|-|\delta|}(n-|\lambda|)_{(|\rho|-|\lambda|)}(n-|\delta|)_{(|\rho|-|\delta|)}},
$$

and

$$
c_{\lambda \delta}^{\rho}(n+1)=\frac{\left|H_{\lambda \delta}^{\rho}(n+1)\right|}{2^{2|\rho|-|\lambda|-|\delta|}(n+1-|\lambda|)_{(|\rho|-|\lambda|)}(n+1-|\delta|)_{(|\rho|-|\delta|)}} .
$$

This gives us after simplification:

$$
\frac{c_{\lambda \delta}^{\rho}(n+1)}{c_{\lambda \delta}^{\rho}(n)}=\frac{\left|H_{\lambda \delta}^{\rho}(n+1)\right|}{\left|H_{\lambda \delta}^{\rho}(n)\right|} \cdot \frac{n+1-|\rho|}{n+1-|\lambda|} \cdot \frac{n+1-|\rho|}{n+1-|\delta|}
$$

We will now evaluate the quotient $\frac{\left|H_{\lambda \delta}^{\rho}(n+1)\right|}{\left|H_{\lambda \delta}^{\rho}(n)\right|}$. Let $\boldsymbol{u}=\left(u_{1}, u_{1}^{\prime}, u_{2}, u_{2}^{\prime}\right)$ be an element of $\mathbf{P}_{n}^{4}$ such that:

$$
\begin{aligned}
& u_{2} \subseteq d, \\
& u_{1}^{\prime} \subseteq d^{\prime}, \\
& \left|u_{1}\right|=\left|u_{1}^{\prime}\right|=2|\lambda|, \\
& \left|u_{2}\right|=\left|u_{2}^{\prime}\right|=2|\delta|, \\
& \left|u_{2}^{\prime} \cup u_{1}\right|=2|\rho| .
\end{aligned}
$$


We introduce

$$
\begin{aligned}
N_{\boldsymbol{u}}= & \left\{\left(h_{1}=\left(f_{1}, u_{1}, u_{1}^{\prime}\right), h_{2}=\left(f_{2}, u_{2}, u_{2}^{\prime}\right)\right) \in A_{\lambda, n} \times A_{\delta, n}\right. \\
& \text { such that there exists } \left.\left(\widetilde{h_{1}}, \widetilde{h_{2}}\right) \in E_{h_{1}}^{h_{2}}(n) \text { with } \alpha=\widetilde{h_{1}} \circ \widetilde{h_{2}}\right\} .
\end{aligned}
$$

Its elements are represented on Figure 8. The set $H_{\lambda \delta}^{\rho}(n)$ is the disjoint union of all $N_{\boldsymbol{u}}$ with $\boldsymbol{u}$ satisfying the above conditions.

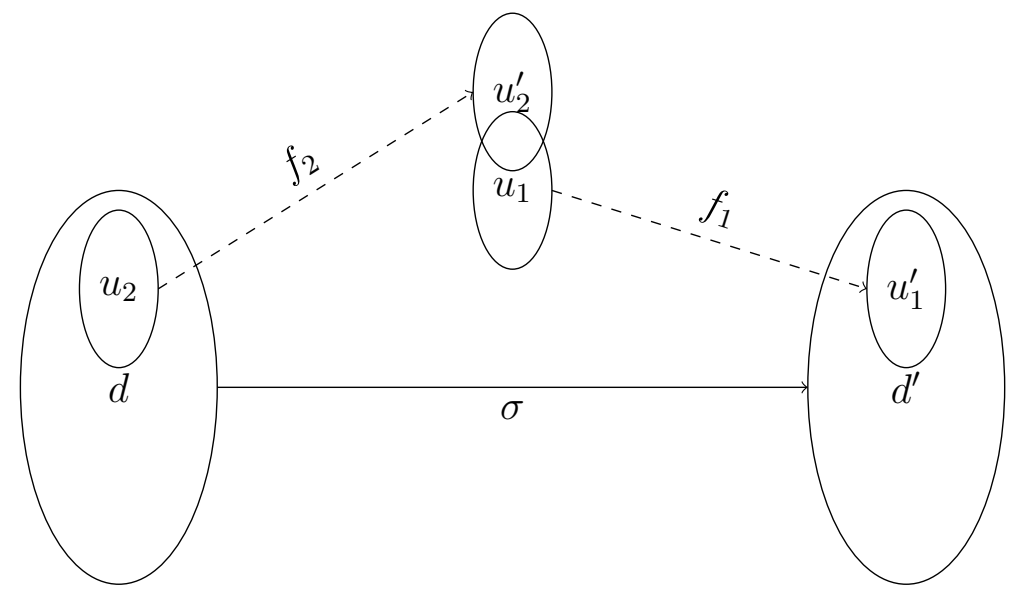

Figure 8: Schematic representation of elements of $N_{\boldsymbol{u}}$

Lemma 3.12. Let $\boldsymbol{v}=\left(v_{1}, v_{1}^{\prime}, v_{2}, v_{2}^{\prime}\right)$ be an element of $\mathbf{P}_{n}^{4}$ satisfying conditions above. If $v_{1}^{\prime}=u_{1}^{\prime}$ and $v_{2}=u_{2}$, then there exists a bijection between $N_{\boldsymbol{u}}$ and $N_{\boldsymbol{v}}$.

Proof. We take any permutation $b \in \mathcal{B}_{n}$, such that $b\left(u_{1}\right)=v_{1}$ and $b\left(u_{2}^{\prime}\right)=v_{2}^{\prime}$. Such a permutation exists because $\left|u_{1}\right|=\left|v_{1}\right|,\left|u_{2}^{\prime}\right|=\left|v_{2}^{\prime}\right|$ and $\left|u_{2}^{\prime} \cup u_{1}\right|=\left|v_{2}^{\prime} \cup v_{1}\right|$. We associate to a pair $\left(h_{1}, h_{2}\right)$ in $N_{\boldsymbol{u}}$ the pair $\left((i d, b) \bullet h_{1},(b, i d) \bullet h_{2}\right)$. We check that the image lies in $N_{v}$ :

$$
\begin{aligned}
\left((i d, b) \bullet h_{1},(b, i d) \bullet h_{2}\right) & =\left(\left(f_{1} b^{-1}, b\left(u_{1}\right), u_{1}^{\prime}\right),\left(b f_{2}, u_{2}, b\left(u_{2}^{\prime}\right)\right)\right) \\
& =\left(\left(f_{1} b^{-1}, v_{1}, u_{1}^{\prime}\right),\left(b f_{2}, u_{2}, v_{2}^{\prime}\right)\right) \in A_{\lambda, n} \times A_{\delta, n} .
\end{aligned}
$$

We can check easily that $\left((i d, b) \bullet \widetilde{h_{1}},(b, i d) \bullet \widetilde{h_{2}}\right) \in E_{(i d, b) \bullet h_{1}}^{(b, i d) \bullet h_{2}}(n)$, and we have:

$$
\begin{aligned}
(i d, b) \bullet \widetilde{h_{1}} \circ(b, i d) \bullet \widetilde{h_{2}} & =\left(\widetilde{f_{1}} b^{-1}, b\left(\widetilde{u_{1}}\right), \widetilde{u_{1}^{\prime}}\right) \circ\left(b \widetilde{f_{2}}, \widetilde{u_{2}}, b\left(\widetilde{u_{2}^{\prime}}\right)\right) \\
& =\left(\widetilde{f_{1}} b^{-1} b \widetilde{f_{2}}, \widetilde{u_{2}}, \widetilde{u_{1}^{\prime}}\right) \\
& =\widetilde{h_{1}} \circ \widetilde{h_{2}} \\
& =\alpha .
\end{aligned}
$$

It is then easy to check that this defines a bijection between $N_{\boldsymbol{u}}$ and $N_{\boldsymbol{v}}$. Details are the same as in Lemma 3.6. 
Lemma 3.12 implies that the cardinality of $N_{\boldsymbol{u}}$ depends only on $u_{1}^{\prime}$ and $u_{2}$. We denote it by $f\left(u_{1}^{\prime}, u_{2}\right)$. If we denote by $\boldsymbol{U}$ the set of vectors $\boldsymbol{u} \in \mathbf{P}_{n}^{4}$ satisfying conditions (13) to (17), the set $H_{\lambda \delta}^{\rho}(n)$ can be written as follows:

$$
H_{\lambda \delta}^{\rho}(n)=\bigsqcup_{\boldsymbol{u} \in \boldsymbol{U}} N_{\boldsymbol{u}}
$$

Using Lemma 3.12, we obtain:

$$
\left|H_{\lambda \delta}^{\rho}(n)\right|=\sum_{\boldsymbol{u} \in \boldsymbol{U}}\left|N_{\boldsymbol{u}}\right|=\sum_{u_{1}^{\prime}, u_{2}} \sum_{u_{1}, u_{2}^{\prime}} f\left(u_{1}^{\prime}, u_{2}\right) .
$$

The first (resp. second) summation indexes are vectors $u_{1}^{\prime}$ and $u_{2}$ (resp. $u_{1}$ and $u_{2}^{\prime}$ ) satisfying conditions (13) to (16) (resp. (15) to (17)). Since $N_{\boldsymbol{u}}$ depends only on $u_{1}^{\prime}$ and $u_{2}$, we get:

$$
\left|H_{\lambda \delta}^{\rho}(n)\right|=\sum_{u_{1}^{\prime}, u_{2}} f\left(u_{1}^{\prime}, u_{2}\right) k_{n}
$$

where $k_{n}$ is the number of possible choices of vectors $u_{1}$ and $u_{2}^{\prime}$ satisfying conditions (15) to (17). There are $\left(\begin{array}{c}n \\ |\lambda|\end{array}\right)$ sets $u_{1} \in \mathbf{P}_{n}$ that fulfill (15). Once $u_{1}$ is chosen, it remains $\left(\begin{array}{c}|\lambda| \\ |\lambda|+|\delta|-|\rho|\end{array}\right) \cdot\left(\begin{array}{c}n-|\lambda| \\ |\rho|-|\lambda|\end{array}\right)$ ways to choose $u_{2}^{\prime}$ with conditions (16) and (17). The first binomial is the number of possible choices of $u_{1} \cap u_{2}^{\prime}$ and the second one is the number of possible choices of $u_{2}^{\prime} \backslash u_{1}$. Then, we have:

$$
k_{n}=\left(\begin{array}{c}
n \\
|\lambda|
\end{array}\right) \cdot\left(\begin{array}{c}
n-|\lambda| \\
|\rho|-|\lambda|
\end{array}\right) \cdot\left(\begin{array}{c}
|\lambda| \\
|\lambda|+|\delta|-|\rho|
\end{array}\right) .
$$

Thus, the cardinality of $H_{\lambda \delta}^{\rho}(n)$ is:

$$
\left|H_{\lambda \delta}^{\rho}(n)\right|=\left(\begin{array}{c}
n \\
|\lambda|
\end{array}\right) \cdot\left(\begin{array}{c}
n-|\lambda| \\
|\rho|-|\lambda|
\end{array}\right) \cdot\left(\begin{array}{c}
|\lambda| \\
|\lambda|+|\delta|-|\rho|
\end{array}\right) \sum_{u_{1}^{\prime}, u_{2}} f\left(u_{1}^{\prime}, u_{2}\right) .
$$

The summation index does not depend on $n$ because $u_{1}^{\prime}$ and $u_{2}$ fulfil conditions (13) and (14). Similarly, we obtain:

$$
\left|H_{\lambda \delta}^{\rho}(n+1)\right|=\left(\begin{array}{c}
n+1 \\
|\lambda|
\end{array}\right) \cdot\left(\begin{array}{c}
n+1-|\lambda| \\
|\rho|-|\lambda|
\end{array}\right) \cdot\left(\begin{array}{c}
|\lambda| \\
|\lambda|+|\delta|-|\rho|
\end{array}\right) \sum_{u_{1}^{\prime}, u_{2}} f\left(u_{1}^{\prime}, u_{2}\right),
$$

which gives us:

$$
\frac{\left|H_{\lambda \delta}^{\rho}(n+1)\right|}{\left|H_{\lambda \delta}^{\rho}(n)\right|}=\frac{\left(\begin{array}{c}
n+1 \\
|\delta|
\end{array}\right)\left(\begin{array}{c}
n+1-|\delta| \\
|\rho|-|\delta|
\end{array}\right)}{\left(\begin{array}{c}
n \\
|\delta|
\end{array}\right)\left(\begin{array}{c}
n-|\delta| \\
|\rho|-|\delta|
\end{array}\right)}=\frac{n+1}{n+1-|\rho|}
$$


Thus, we have:

$$
\begin{aligned}
\frac{c_{\lambda \delta}^{\rho}(n+1)}{c_{\lambda \delta}^{\rho}(n)} & =\frac{n+1}{n+1-|\rho|} \cdot \frac{n+1-|\rho|}{n+1-|\lambda|} \cdot \frac{n+1-|\rho|}{n+1-|\delta|} \\
& =\frac{\frac{n+1}{(n+1-|\lambda|)} \frac{n+1}{(n+1-|\delta|)}}{\frac{n+1}{(n+1-|\rho|)}} .
\end{aligned}
$$

This proves that $\varphi_{n}$ is a homomorphism of algebras.

\subsection{Projective limits}

In this subsection, we consider the projective limit $\mathcal{A}_{\infty}$ of the sequence $\left(\mathcal{A}_{n}\right)$. We prove in Proposition 3.15 that every element of $\mathcal{A}_{\infty}$ is written in a unique way as infinite linear combination of elements indexed by partitions.

From equation (18), we get the following corollary.

Corollary 3.13. Let $\lambda, \delta$ and $\rho$ be three partitions such that

$$
\max (|\lambda|,|\delta|) \leqslant|\rho| \leqslant|\lambda|+|\delta| .
$$

For every $n \geqslant|\rho|$, we have:

$$
c_{\lambda \delta}^{\rho}(n)=\frac{c_{\lambda \delta}^{\rho}(|\rho|)}{\left(\begin{array}{c}
|\rho| \\
|\lambda|
\end{array}\right)\left(\begin{array}{c}
|\rho| \\
|\delta|
\end{array}\right)} \cdot \frac{\left(\begin{array}{c}
n \\
|\lambda|
\end{array}\right)\left(\begin{array}{c}
n \\
|\delta|
\end{array}\right)}{\left(\begin{array}{c}
n \\
|\rho|
\end{array}\right)} .
$$

Proof. We proceed by induction on $n$. For $n=|\rho|$, we have the equality. Assume we have the equality for some $n \geqslant|\rho|$ and let us prove it for $n+1$. By equation (18), we have:

$$
\frac{c_{\lambda \delta}^{\rho}(n+1)}{c_{\lambda \delta}^{\rho}(n)}=\frac{\frac{n+1}{(n+1-|\lambda|)} \frac{n+1}{(n+1-|\delta|)}}{\frac{n+1}{(n+1-|\rho|)}} .
$$

This gives us the following equality, using the induction hypothesis :

$$
\begin{aligned}
c_{\lambda \delta}^{\rho}(n+1) & =\frac{c_{\lambda \delta}^{\rho}(|\rho|)}{\left(\begin{array}{c}
|\rho| \\
|\lambda|
\end{array}\right)\left(\begin{array}{c}
|\rho| \\
|\delta|
\end{array}\right)} \cdot \frac{\left(\begin{array}{c}
n \\
|\lambda|
\end{array}\right)\left(\begin{array}{c}
n \\
|\delta|
\end{array}\right)}{\left(\begin{array}{c}
n \\
|\rho|
\end{array}\right)} \cdot \frac{\frac{n+1}{(n+1-|\lambda|)} \frac{n+1}{(n+1-|\delta|)}}{\frac{n+1}{(n+1-|\rho|)}} \\
& =\frac{c_{\lambda \delta}^{\rho}(|\rho|)}{\left(\begin{array}{c}
|\rho| \\
|\lambda|
\end{array}\right)\left(\begin{array}{l}
|\rho| \\
|\delta|
\end{array}\right)} \cdot \frac{\left(\begin{array}{c}
n+1 \\
|\lambda|
\end{array}\right)\left(\begin{array}{c}
n+1 \\
|\delta|
\end{array}\right)}{\left(\begin{array}{c}
n+1 \\
|\rho|
\end{array}\right)} .
\end{aligned}
$$


Let $\mathcal{A}_{\infty}$ be the projective limit of $\left(\mathcal{A}_{n}, \varphi_{n}\right)$ :

$$
\mathcal{A}_{\infty}=\left\{\left(a_{n}\right)_{n \geqslant 1} \mid \text { for every } n \geqslant 1, a_{n} \in \mathcal{A}_{n} \text { and } \varphi_{n}\left(a_{n+1}\right)=a_{n}\right\} .
$$

Lemma 3.14. Elements of $\mathcal{A}_{\infty}$ are of the form $a=\left(a_{n}\right)_{n \geqslant 1}$, where

$$
a_{n}=\sum_{\substack{\lambda \text { partition } \\
|\lambda| \leqslant n}} \frac{x_{\lambda}^{a}}{\left(\begin{array}{c}
n \\
|\lambda|
\end{array}\right)} S_{\lambda, n}
$$

for some sequence $x=\left(x_{\lambda}^{a}\right)_{\lambda \text { partition }}$ of scalars uniquely determined by $a$.

Proof. Let $a=\left(a_{n}\right)_{n \geqslant 1}$ be a sequence in $\mathcal{A}_{\infty}, a_{n} \in \mathcal{A}_{n}$ for every $n \geqslant 1$. By Proposition 3.8 , the elements $\left(S_{\lambda, n}\right)_{\lambda \vdash r \leqslant n}$ form a basis of $\mathcal{A}_{n}$, thus for every $n \geqslant 1$ and every partition $\lambda$ such as $|\lambda| \leqslant n$, there exists a scalar $a_{\lambda}(n) \in \mathbb{C}$ such that

$$
a_{n}=\sum_{\substack{\lambda \text { partition } \\|\lambda| \leqslant n}} a_{\lambda}(n) S_{\lambda, n} .
$$

The condition $\varphi_{n}\left(a_{n+1}\right)=a_{n}$ can be written as follows:

$$
\varphi_{n}\left(\sum_{\substack{\lambda \text { partition } \\|\lambda| \leqslant n+1}} a_{\lambda}(n+1) S_{\lambda, n+1}\right)=\sum_{\substack{\lambda \text { partition } \\|\lambda| \leqslant n}} a_{\lambda}(n) S_{\lambda, n} .
$$

Using the definition of $\varphi_{n}$, we simplify this equality to obtain:

$$
\sum_{\substack{\lambda \text { partition } \\|\lambda| \leqslant n}} a_{\lambda}(n+1) \frac{n+1}{n+1-|\lambda|} S_{\lambda, n}=\sum_{\substack{\lambda \text { partition } \\|\lambda| \leqslant n}} a_{\lambda}(n) S_{\lambda, n} .
$$

By comparing the coefficients of $S_{\lambda, n}$ we get that for every partition $\lambda$ such that $|\lambda| \leqslant n$, we have:

$$
\frac{a_{\lambda}(n+1)}{a_{\lambda}(n)}=\frac{n+1-|\lambda|}{n+1} .
$$

By induction, we get :

$$
a_{\lambda}(n)=\frac{a_{\lambda}(|\lambda|)}{\left(\begin{array}{c}
n \\
|\lambda|
\end{array}\right)}
$$

We set $x_{\lambda}^{a}=a_{\lambda}(|\lambda|)$. This proves Lemma 3.14.

For every partition $\lambda$, we define the sequence $T_{\lambda}:=\left(T_{\lambda, n}\right)_{n \geqslant 1}$ where :

$$
T_{\lambda, n}= \begin{cases}0 & \text { if } n<|\lambda|, \\
\frac{1}{\left(\begin{array}{c}
n \\
|\lambda|
\end{array}\right)} S_{\lambda, n} & \text { if } n \geqslant|\lambda| .\end{cases}
$$

From Lemma 3.14, we obtain directly the following proposition: 
Proposition 3.15. Every element $a \in \mathcal{A}_{\infty}$ is written in a unique way as infinite linear combination of elements $T_{\lambda}$.

This proposition shows that the algebra $\mathcal{A}_{\infty}$ satisfies the second property required in Section 2.5. In particular, $T_{\lambda} * T_{\delta}$ writes as linear combination of elements $T_{\rho}$. We can be more precise.

Corollary 3.16. Let $\lambda$ and $\delta$ be two partitions, there exist unique constants $b_{\lambda \delta}^{\rho}$ such that:

$$
T_{\lambda} * T_{\delta}=\sum_{\substack{\rho \text { partition } \\ \max (|\lambda|,|\delta|) \leqslant|\rho| \leqslant|\lambda|+|\delta|}} b_{\lambda \delta}^{\rho} T_{\rho}
$$

Moreover $b_{\lambda \delta}^{\rho}=\frac{c_{\lambda \delta}^{\rho}(|\rho|)}{\left(\begin{array}{l}|\rho| \\ |\lambda|\end{array}\right)\left(\begin{array}{l}|\rho| \\ |\delta|\end{array}\right)}$. In particular, it is a non-negative rational number.

Proof. By Proposition 3.15, $T_{\lambda} * T_{\delta}$ may be written as a linear combination of elements $T_{\rho}$ :

$$
T_{\lambda} * T_{\delta}=\sum_{\rho \text { partition }} b_{\lambda \delta}^{\rho} T_{\rho} .
$$

It remains to prove the conditions about the size of partitions $\rho$ that appear in the sum index and the formula for $b_{\lambda \delta}^{\rho}$.

If $n<\max (|\lambda|,|\delta|)$, we have:

$$
\left(T_{\lambda} * T_{\delta}\right)_{n}=0 .
$$

Let $n \geqslant \max (|\lambda|,|\delta|)$, we use Corollary 3.9 and Corollary 3.13 to get:

$$
\begin{aligned}
\left(T_{\lambda} * T_{\delta}\right)_{n} & =\frac{1}{\left(\begin{array}{c}
n \\
|\lambda|
\end{array}\right)} S_{\lambda, n} * \frac{1}{\left(\begin{array}{c}
n \\
|\delta|
\end{array}\right)} S_{\delta, n} \\
& =\sum_{\substack{\rho \text { partition } \\
\max (|| \lambda|,| \delta \mid) \leqslant|\rho| \leqslant \min (|\lambda|+|\delta|, n)}} \frac{c_{\lambda \delta}^{\rho}(|\rho|)}{\left(\begin{array}{l}
|\rho| \\
|\lambda|
\end{array}\right)\left(\begin{array}{l}
|\rho| \\
|\delta|
\end{array}\right)\left(\begin{array}{c}
n \\
|\rho|
\end{array}\right)} S_{\rho, n} \\
& =\left(\sum_{\substack{\rho \text { partition } \\
\max (|\lambda|,|\delta|) \leqslant|\rho| \leqslant|\lambda|+|\delta|}} \frac{c_{\lambda \delta}^{\rho}(|\rho|)}{\left(\begin{array}{l}
|\rho| \\
|\lambda|
\end{array}\right)\left(\begin{array}{l}
|\rho| \\
|\delta|
\end{array}\right)} T_{\rho}\right)_{n}
\end{aligned}
$$

Comparing both expressions for $T_{\lambda} * T_{\delta}$, this proves our proposition. 
Example 3.5. We compute in this example the product $T_{(2)} * T_{(2)}$. Using Corollary 3.16, we can write $T_{(2)} * T_{(2)}=\sum_{\substack{\rho \text { partition } \\ 2 \leqslant|\rho| \leqslant 4}} b_{(2)(2)}^{\rho} T_{\rho}$, which gives us:

$$
\begin{aligned}
T_{(2)} * T_{(2)}= & b_{(2)(2)}^{\left(1^{2}\right)} T_{\left(1^{2}\right)}+b_{(2)(2)}^{\left(1^{3}\right)} T_{\left(1^{3}\right)}+b_{(2)(2)}^{\left(1^{4}\right)} T_{\left(1^{4}\right)} \\
& +b_{(2)(2)}^{(2)} T_{(2)}+b_{(2)(2)}^{(2,1)} T_{(2,1)}+b_{(2)(2)}^{\left(2,1^{2}\right)} T_{\left(2,1^{2}\right)}+b_{(2)(2)}^{\left(2^{2}\right)} T_{\left(2^{2}\right)} \\
& +b_{(2)(2)}^{(3)} T_{(3)}+b_{(2)(2)}^{(3,1)} T_{(3,1)} \\
& +b_{(2)(2)}^{(4)} T_{(4)} .
\end{aligned}
$$

The formula for $b_{\lambda \delta}^{\rho}$ given in Corollary 3.16 shows that these elements can be computed using the product of $S_{(2),|\lambda|+|\delta|} * S_{(2),|\lambda|+|\delta|}$ in $\mathcal{A}_{|\lambda|+|\delta|}$, which is $\mathcal{A}_{4}$ in our case.

We have implemented the algebra $\mathcal{A}_{n}$ in [Sag] and got the following equation for the product $S_{(2), 4} * S_{(2), 4}$ in $\mathcal{A}_{4}$ :

$$
S_{(2), 4} * S_{(2), 4}=96 S_{\left(1^{2}\right), 4}+48 S_{(2), 4}+36 S_{(3), 4}+12 S_{\left(2^{2}\right), 4}
$$

Using the formulas for $c_{\lambda \delta}^{\rho}(|\rho|)$ and $b_{\lambda \delta}^{\rho}$ given in Corollary 3.13 and 3.16, we obtain:

$$
T_{(2)} * T_{(2)}=16 T_{\left(1^{2}\right)}+8 T_{(2)}+4 T_{(3)}+\frac{1}{3} T_{\left(2^{2}\right)} .
$$

Corollary 3.17. The set of all finite linear combinations of $\left(T_{\lambda}\right)$, denoted by $\widetilde{\mathcal{A}_{\infty}}$, forms a sub-algebra of $\mathcal{A}_{\infty}$. The family $\left(T_{\lambda}\right)_{\lambda}$ partition is a basis of $\widetilde{\mathcal{A}_{\infty}}$.

Proof. This comes from the fact that the partitions $\rho$ indexing the sum in the product $T_{\lambda} * T_{\delta}$ satisfy :

$$
|\rho| \leqslant|\lambda|+|\delta|
$$

The algebra $\widetilde{\mathcal{A}_{\infty}}$ will be of interest in Section 5 .

\section{Proof of Theorem 2.1}

In the previous section, we built all algebras and homomorphisms that we need in order to prove Theorem 2.1 .

Let $\lambda$ and $\delta$ be two proper partitions, by Corollary 3.16, we have:

$$
T_{\lambda} * T_{\delta}=\sum_{\substack{\rho \text { partition } \\ \max (|\lambda|,|\delta|) \leqslant|\rho| \leqslant|\lambda|+|\delta|}} b_{\lambda \delta}^{\rho} T_{\rho} .
$$

Recall that this is an equality of sequences. Taking the $n$-th term, we have:

$$
\frac{1}{\left(\begin{array}{c}
n \\
|\lambda|
\end{array}\right)} S_{\lambda, n} * \frac{1}{\left(\begin{array}{c}
n \\
|\delta|
\end{array}\right)} S_{\delta, n}=\sum_{\substack{\rho \text { partition } \\
\max (|\lambda|,|\delta| \leqslant|\rho| \leqslant \min (|\lambda|+|\delta|, n)}} b_{\lambda \delta}^{\rho} \frac{1}{\left(\begin{array}{c}
n \\
|\rho|
\end{array}\right)} S_{\rho, n} .
$$


By applying $\psi_{n}$ we obtain (see Lemma 3.10):

$$
\begin{aligned}
\frac{1}{2^{n-|\lambda|}(n-|\lambda|) !} K_{\lambda}(n) \cdot \frac{1}{2^{n-|\delta|}(n-|\delta|) !} K_{\delta}(n)= & \\
\sum_{\substack{\rho \text { partition } \\
\max (|\lambda|,|\delta|) \leqslant|\rho| \leqslant \min (|\lambda|+|\delta|, n)}} b_{\lambda \delta}^{\rho} \frac{\left(\begin{array}{c}
n \\
|\lambda|
\end{array}\right)\left(\begin{array}{c}
n \\
|\delta|
\end{array}\right)}{\left(\begin{array}{c}
n \\
|\rho|
\end{array}\right) 2^{n-|\rho|}(n-|\rho|) !} & \left(\begin{array}{c}
n-|\bar{\rho}| \\
m_{1}(\rho)
\end{array}\right) K_{\bar{\rho}}(n) .
\end{aligned}
$$

After simplification, we get:

$$
K_{\lambda}(n) \cdot K_{\delta}(n)=\sum_{\substack{\rho \text { partition } \\ \max (|\lambda|,|\delta|) \leqslant|\rho| \leqslant \min (|\lambda|+|\delta|, n)}} b_{\lambda \delta}^{\rho} \frac{(|\rho|)|\bar{\rho}|}{|\lambda| !|\delta| !} 2^{n+|\rho|-|\lambda|-|\delta|} n !(n-|\bar{\rho}|)_{m_{1}(\rho)} K_{\bar{\rho}}(n) .
$$

Fact. Any partition $\rho$ such that $|\rho| \leqslant \min (|\lambda|+|\delta|, n)$ can be written in a unique way as $\rho=\tau \cup\left(1^{j}\right)$, where $\tau$ is a proper partition and $j \leqslant \min (|\lambda|+|\delta|, n)-|\tau|$.

Using this fact, the product can be written as follows:

$$
K_{\lambda}(n) \cdot K_{\delta}(n)=\sum_{\substack{\tau \text { proper partition } \\|\tau| \leqslant \min (|\lambda|+|\delta|, n)}} \alpha_{\lambda \delta}^{\tau}(n) K_{\tau}(n),
$$

where

$$
\begin{aligned}
\alpha_{\lambda \delta}^{\tau}(n) & =\frac{1}{|\lambda| !|\delta| !} \sum_{j=0}^{\min (|\lambda|+|\delta|, n)-|\tau|} b_{\lambda \delta}^{\tau \cup\left(1^{j}\right)} n !(n-|\tau|)_{j}(|\tau|+j)_{|\tau|} 2^{n+|\tau|+j-|\lambda|-|\delta|} \\
& =\left.\frac{2^{n} n !}{|\lambda| !|\delta| !} \sum_{j=0}^{|\lambda|+|\delta|-|\tau|} b_{\lambda \delta}^{\tau \cup\left(1^{j}\right)}(n-|\tau|)_{j}(|\tau|+j)_{|\tau|}\right|^{|\tau|+j-|\lambda|-|\delta|} .
\end{aligned}
$$

The change of sum index in the last equality comes from the fact that if $n<|\lambda|+|\delta|$, we have:

$$
(n-|\tau|)_{j}=0 \text { for any } j \text { with } n-|\tau|<j \leqslant|\lambda|+|\delta|-|\tau| .
$$

This ends the proof of Theorem 2.1.

Corollary 4.1. If $\lambda, \delta$ and $\rho$ are three proper partitions such that $|\rho|=|\lambda|+|\delta|$, then:

$$
\alpha_{\lambda \delta}^{\rho}(n)=b_{\lambda \delta}^{\rho} \frac{|\rho| !}{|\lambda| !|\delta| !} 2^{n} n !=c_{\lambda \delta}^{\rho}(|\rho|) \frac{|\lambda| !|\delta| !}{(|\lambda|+|\delta|) !} 2^{n} n !
$$

Example 4.1. We recall that the product $T_{(2)} * T_{(2)}$ has been computed in Example 3.5. We deduce from it the complete formula for the product $K_{(2)}(n) \cdot K_{(2)}(n)$ for every $n \geqslant 4$. Using formula (19), we have:

$$
K_{(2)}(n) \cdot K_{(2)}(n)=2^{n} n ! n(n-1) K_{\emptyset}(n)+2^{n} n ! K_{(2)}(n)+2^{n} n ! 3 K_{(3)}(n)+2^{n} n ! 2 K_{\left(2^{2}\right)}(n) .
$$




\section{$5 \quad$ A link with shifted symmetric functions}

In [IK99, Section 9], Ivanov and Kerov have given an isomorphism between the algebra of 1-shifted symmetric functions and the algebra $\mathbf{A}_{\infty}$, which is the universal algebra that projects on the center of the symmetric group algebra $Z\left(\mathbb{C}\left[\mathcal{S}_{n}\right]\right)$, for each $n$. In this section, using the zonal spherical functions of the Gelfand pair $\left(\mathcal{S}_{2 n}, \mathcal{B}_{n}\right)$, we prove that there is an isomorphism between the algebra of 2-shifted symmetric functions and the algebra $\widetilde{\mathcal{A}_{\infty}}$.

We start with the definition of the algebra of shifted symmetric functions with coefficients in $\mathbb{C}(\alpha)$, denoted by $\Lambda^{*}(\alpha)$. An $\alpha$-shifted symmetric function $f$ in infinitely many variables $\left(x_{1}, x_{2}, \cdots\right)$ is a family $\left(f_{i}\right)_{i \geqslant 1}$ with the two following properties:

1. $f_{i}$ is a symmetric polynomial in $\left(x_{1}-\frac{1}{\alpha}, x_{2}-\frac{2}{\alpha}, \cdots, x_{i}-\frac{i}{\alpha}\right)$.

2. $f_{i+1}\left(x_{1}, x_{2}, \cdots, x_{i}, 0\right)=f_{i}\left(x_{1}, x_{2}, \cdots, x_{i}\right)$.

The set of all shifted symmetric functions is an algebra denoted $\Lambda^{*}(\alpha)$. In [Las08], Lassalle gives an isomorphism between the algebra of symmetric functions with coefficients in $\mathbb{C}[\alpha]$, denoted by $\Lambda(\alpha)$, and $\Lambda^{*}(\alpha)$. We will denote this isomorphism by $s h_{\alpha}$ instead of $(\#)$, as used by Lassalle. We prefer this notation as it makes the dependence in the parameter $\alpha$ explicit.

Let $f$ be an element of $\Lambda^{*}(\alpha)$. For any partition $\lambda=\left(\lambda_{1}, \lambda_{2}, \cdots, \lambda_{l}\right)$, we denote by $f(\lambda)$ the value $f_{l}\left(\lambda_{1}, \lambda_{2}, \cdots, \lambda_{l}\right)$. The shifted symmetric function $f$ is determined by its values on partitions, see [OO97, Section 2].

\subsection{Gelfand pairs and zonal spherical functions}

Let $G$ be a finite group and $K$ a subgroup of $G$. We denote by $C(G, K)$ the set of all complex-valued functions on $G$ that are constant on each $K$-double coset in $G$. Namely,

$$
C(G, K)=\left\{f: G \longrightarrow \mathbb{C} \text { such that } f\left(k x k^{\prime}\right)=f(x) \text { for all } x \text { in } G \text { and } k, k^{\prime} \text { in } K\right\} .
$$

The set $C(G, K)$ is an algebra with product defined as follows (usually called convolution product):

$$
(f g)(x)=\sum_{y \in G} f(y) g\left(y^{-1} x\right) \text { for all } f, g \text { in } C(G, K) .
$$

The pair $(G, K)$ is said to be a Gelfand pair if the algebra $C(G, K)$ is commutative. More details about Gelfand pairs are given in [Mac95, Chapter VII, 1]. In particular, when $(G, K)$ is a Gelfand pair, the algebra $C(G, K)$ admit a relevant canonical basis $\left(\omega_{i}\right)$. The $\omega_{i}$ are called zonal spherical functions.

Proposition 5.1. Every zonal spherical function $\omega$ of a Gelfand pair $(G, K)$ defines a homomorphism of $\mathbb{C}[K \backslash G / K]$ to $\mathbb{C}^{*}$, where $\mathbb{C}[K \backslash G / K]$ is the sub-algebra of $\mathbb{C}[G]$ of elements invariant under the $K$-double action. 
Proof. A zonal spherical function $\omega$ has the following property given in [Mac95, page 392]:

$$
\omega(x) \omega(y)=\frac{1}{|K|} \sum_{k \in K} \omega(x k y),
$$

for all $x, y \in G$. This property can be extended by linearity to the group algebra $\mathbb{C}[G]$. If $x$ and $y$ are two elements of $\mathbb{C}[K \backslash G / K]$, then we have:

$$
\omega(x) \omega(y)=\frac{1}{|K|} \sum_{k \in K} \omega(x k y)=\frac{1}{|K|} \sum_{k \in K} \omega(x y)=\omega(x y),
$$

which ends the proof of the proposition.

The pair $\left(\mathcal{S}_{2 n}, \mathcal{B}_{n}\right)$ is a Gelfand pair (see [Mac95, Chapter VII, 2]) and its zonal spherical functions are indexed by partitions of $n$. They are denoted by $\omega^{\rho}$ and defined by:

$$
\omega^{\rho}(x)=\frac{1}{\left|\mathcal{B}_{n}\right|} \sum_{k \in \mathcal{B}_{n}} \chi^{2 \rho}(x k),
$$

for $x \in \mathcal{S}_{2 n}$, where $\chi^{2 \rho}$ is the character of the irreducible $\mathcal{S}_{2 n}$-module corresponding to $2 \rho:=\left(2 \rho_{1}, 2 \rho_{2}, \cdots\right)$. Two permutations $x$ and $y$ in the same $\mathcal{B}_{n}$-double coset $K_{\lambda}(n)$ have the same image by $\omega^{\rho}$ denoted by $\omega_{\lambda}^{\rho}$.

\section{$5.2 \quad$ Jack polynomials}

The family of Jack polynomials $J_{\rho}(\alpha)$, indexed by partitions, forms a basis of $\Lambda(\alpha)$. In the basis of power sums $p_{\lambda}, J_{\rho}(\alpha)$ may be developed as follows:

$$
J_{\rho}(\alpha)=\sum_{|\lambda|=|\rho|} \theta_{\lambda}^{\rho}(\alpha) p_{\lambda} .
$$

Let $\lambda$ and $\rho$ be partitions with $|\rho| \geqslant|\lambda|$. Then the shifted symmetric function $s h_{\alpha}\left(p_{\lambda}\right)$ is related to $\theta_{\lambda \cup\left(1^{n-|\lambda|}\right)}^{\rho}(\alpha)$ by the following equation given in [Las08, Proposition 2]:

$$
\alpha^{|\lambda|-l(\lambda)} \operatorname{sh}_{\alpha}\left(p_{\lambda}\right)(\rho)=\left(\begin{array}{c}
|\rho|-|\lambda|+m_{1}(\lambda) \\
m_{1}(\lambda)
\end{array}\right) z_{\lambda} \theta_{\lambda \cup\left(1^{n-|\lambda|}\right)}^{\rho}(\alpha)
$$

where $z_{\lambda}=\prod_{i \geqslant 1} i^{m_{i}(\lambda)} m_{i}(\lambda)$ !. Directly from the definition of $s h_{\alpha}(f)$ for any symmetric function $f$, given in [Las08, Eq. (3.1)], one has $s h_{\alpha}\left(p_{\lambda}\right)(\rho)=0$ if $|\rho|<|\lambda|$.

\subsection{Isomorphism between $\widetilde{\mathcal{A}_{\infty}}$ and $\Lambda^{*}(2)$}

When $\alpha=2$, Jack polynomials are related to zonal spherical functions of $\left(\mathcal{S}_{2 n}, \mathcal{B}_{n}\right)$ by the following equation (cf. [Mac95, page 408]):

$$
J_{\rho}(2)=\left|\mathcal{B}_{n}\right| \sum_{|\lambda|=n} z_{2 \lambda}^{-1} \omega_{\lambda}^{\rho} p_{\lambda}
$$


for every partition $\rho$ of $n$. This formula can be viewed as an analogue for $\alpha=2$ of the following formula known as Frobenius formula, see [Mac95, page 114]:

$$
s_{\rho}=\sum_{|\lambda|=|\rho|} z_{\lambda}^{-1} \chi_{\lambda}^{\rho} p_{\lambda}
$$

where $s_{\rho}$ is the Schur function. Equations (20) and (22) give us the following equality when $\alpha=2$ :

$$
\theta_{\lambda}^{\rho}(2)=\left|\mathcal{B}_{|\rho|}\right| z_{2 \lambda}^{-1} \omega_{\lambda}^{\rho}
$$

Theorem 5.1. The linear mapping $F: \widetilde{\mathcal{A}_{\infty}} \longrightarrow \Lambda^{*}(2)$ defined by:

$$
F\left(T_{\lambda}\right)=2^{2|\lambda|-l(\lambda)}|\lambda| ! \frac{s h_{2}\left(p_{\lambda}\right)}{z_{\lambda}},
$$

is an isomorphism of algebras.

Proof. Let $\lambda$ be a partition and $T_{\lambda}$ the corresponding element in $\widetilde{\mathcal{A}_{\infty}}$. Let $n$ be an integer, $n \geqslant|\lambda|$. By definition, $T_{\lambda}$ is a sequence and its $n$-th term $\left(T_{\lambda}\right)_{n}=\frac{1}{\left(\begin{array}{c}n \\ |\lambda|\end{array}\right)} S_{\lambda, n}$ lies in $\mathcal{A}_{n}$. We project onto $\mathbb{C}\left[\mathcal{B}_{n} \backslash \mathcal{S}_{2 n} / \mathcal{B}_{n}\right]$ by applying $\psi_{n}$. By Lemma 3.10, we get:

$$
\begin{aligned}
\psi_{n}\left(\left(T_{\lambda}\right)_{n}\right) & =\frac{1}{\left(\begin{array}{c}
n \\
|\lambda|
\end{array}\right)} \frac{1}{2^{n-|\lambda|}(n-|\lambda|) !}\left(\begin{array}{c}
n-|\bar{\lambda}| \\
m_{1}(\lambda)
\end{array}\right) K_{\bar{\lambda}}(n) \\
& =\frac{2^{|\lambda|}|\lambda| !}{2^{n} n !}\left(\begin{array}{c}
n-|\bar{\lambda}| \\
m_{1}(\lambda)
\end{array}\right) K_{\bar{\lambda}}(n) .
\end{aligned}
$$

For any partition $\rho$ with size equal to $n$, by applying $\omega^{\rho}$ to $\psi_{n}\left(\left(T_{\lambda}\right)_{n}\right)$, we obtain:

$$
\begin{aligned}
\omega^{\rho}\left(\psi_{n}\left(\left(T_{\lambda}\right)_{n}\right)\right) & =\frac{2^{|\lambda|}|\lambda| !}{2^{n} n !}\left(\begin{array}{c}
n-|\bar{\lambda}| \\
m_{1}(\lambda)
\end{array}\right)\left|K_{\bar{\lambda}}(n)\right| \omega_{\bar{\lambda} \cup\left(1^{n-|\bar{\lambda}|}\right)}^{\rho} \\
& =\frac{2^{|\lambda|}|\lambda| !}{2^{n} n !}\left(\begin{array}{c}
n-|\bar{\lambda}| \\
m_{1}(\lambda)
\end{array}\right)\left|K_{\bar{\lambda}}(n)\right| \theta_{\bar{\lambda} \cup\left(1^{n-|\bar{\lambda}|}\right)}^{\rho}(2) \frac{1}{\left|\mathcal{B}_{n}\right| z_{2\left(\bar{\lambda} \cup\left(1^{n-|\bar{\lambda}|}\right)\right.}^{-1}} .
\end{aligned}
$$

We denote by $\left|K_{\bar{\lambda}}(n)\right|$ the number of permutations of $2 n$ with coset-type $\bar{\lambda} \cup\left(1^{n-|\bar{\lambda}|}\right)$. This number is equal to $\frac{\left|\mathcal{B}_{n}\right|^{2}}{z_{2\left(\bar{\lambda} \cup\left(1^{n-|\bar{\lambda}|}\right)\right.}}$ (see [Mac95, page 402]). Thus, after simplification, we get:

$$
\omega^{\rho}\left(\psi_{n}\left(\left(T_{\lambda}\right)_{n}\right)\right)=2^{|\lambda|}|\lambda| !\left(\begin{array}{c}
n-|\bar{\lambda}| \\
m_{1}(\lambda)
\end{array}\right) \theta_{\bar{\lambda} \cup\left(1^{n-|\bar{\lambda}|}\right)}^{\rho}(2),
$$

which together with (21) gives us the following equation:

$$
\omega^{\rho}\left(\psi_{n}\left(\left(T_{\lambda}\right)_{n}\right)\right)=2^{2|\lambda|-l(\lambda)}|\lambda| ! \frac{s h_{2}\left(p_{\lambda}\right)(\rho)}{z_{\lambda}} .
$$


This formula is valid for any partition $\rho$ such that $|\rho| \geqslant|\lambda|$. We can check that it is also valid for any partition $\rho$ with size less than $|\lambda|$, since in this case $\left(T_{\lambda}\right)_{|\rho|}$ and $s h_{2}\left(p_{\lambda}\right)(\rho)$ are both equal to zero.

Finally, for any partition $\rho$, its image by $F\left(T_{\lambda}\right)$ as defined in the statement of Theorem 5.1, can also be written as follows:

$$
F\left(T_{\lambda}\right)(\rho)=\omega^{\rho}\left(\psi_{|\rho|}\left(\left(T_{\lambda}\right)_{|\rho|}\right)\right) .
$$

Let $\delta$ be a partition, for any partition $\rho$, we have:

$$
\begin{aligned}
F\left(T_{\lambda} * T_{\delta}\right)(\rho) & =\omega^{\rho}\left(\psi_{|\rho|}\left(\left(T_{\lambda} * T_{\delta}\right)_{|\rho|}\right)\right) \\
& =\omega^{\rho}\left(\psi_{|\rho|}\left(\left(T_{\lambda}\right)_{|\rho|} *\left(T_{\delta}\right)_{|\rho|}\right)\right) \\
& =\omega^{\rho}\left(\psi_{|\rho|}\left(T_{\lambda}\right)_{|\rho|} * \psi_{|\rho|}\left(T_{\delta}\right)_{|\rho|}\right) \\
& =\omega^{\rho}\left(\psi_{|\rho|}\left(T_{\lambda}\right)_{|\rho|}\right) \cdot \omega^{\rho}\left(\psi_{|\rho|}\left(T_{\delta}\right)_{|\rho|}\right) .
\end{aligned}
$$

The last equality comes from the fact that $\omega^{\rho}$ defines a homomorphism of $\mathbb{C}\left[\mathcal{B}_{n} \backslash \mathcal{S}_{2 n} / \mathcal{B}_{n}\right]$ to $\mathbb{C}^{*}$ (Proposition 5.1). Hence,

$$
F\left(T_{\lambda} * T_{\delta}\right)(\rho)=\left(F\left(T_{\lambda}\right) \cdot F\left(T_{\delta}\right)\right)(\rho),
$$

for any two partitions $\lambda$ and $\delta$. That means that $F$ is a homomorphism of algebras from $\widetilde{\mathcal{A}_{\infty}}$ to $\Lambda^{*}(2)$. Since $\left(T_{\lambda}\right)_{\lambda \text { partition }}$ and $\left(s h_{2}\left(p_{\lambda}\right)\right)_{\lambda \text { partition }}$ are respectively bases of $\widetilde{\mathcal{A}_{\infty}}$ and $\Lambda^{*}(2), F$ is actually an isomorphism of algebras.

Remark 5.2. The reader should remark while reading the proof that $F\left(T_{\lambda}\right)$ could be defined by $F\left(T_{\lambda}\right)(\rho)=\omega^{\rho}\left(\psi_{|\rho|}\left(\left(T_{\lambda}\right)_{|\rho|}\right)\right)$, which would show directly that $F\left(T_{\lambda}\right)$ is a homomorphism since it is the composition of homomorphisms. However, we prefer to use the definition given by the equation (23) because it gives us explicitly the action of $F$ on the elements of basis of $\widetilde{\mathcal{A}_{\infty}}$.

\subsection{Structure constants}

As said in the beginning of this section, $s h_{\alpha}$ is an isomorphism between $\Lambda(\alpha)$ and $\Lambda^{*}(\alpha)$.

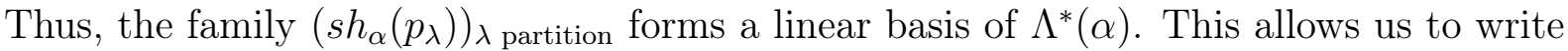
the following equation:

$$
s h_{\alpha}\left(p_{\lambda}\right) s h_{\alpha}\left(p_{\delta}\right)=\sum_{\rho} g_{\lambda, \delta}^{\rho}(\alpha) s h_{\alpha}\left(p_{\rho}\right)
$$

It is proven in [DF14] that the coefficients $g_{\lambda, \delta}^{\rho}(\alpha)$ are polynomial in $\frac{\alpha-1}{\sqrt{\alpha}}$. This structure constants are also related to the Matching-Jack conjecture of Goulden and Jackson, see[DF14, Appendix B].

We are interested in the case $\alpha=2$. We proved in 3.16 that the coefficients $b_{\lambda \delta}^{\rho}$ that appear in the product $T_{\lambda} * T_{\delta}$ are non-negative rational numbers. By applying the isomorphism $F$ given in 5.1 to the product $T_{\lambda} * T_{\delta}$, we get directly the following proposition.

Proposition 5.2. The coefficients $g_{\lambda, \delta}^{\rho}(2)$ are non-negative rational numbers. 


\section{$6 \quad$ Filtrations of the algebra $\mathcal{A}_{\infty}$}

We gave in Theorem 2.1 a polynomiality property of the structure coefficients of the Hecke algebra of the pair $\left(\mathcal{S}_{2 n}, \mathcal{B}_{n}\right)$. The aim of this section is to give upper bounds for the degree of these polynomials. To do this, we study in this section some filtrations of the algebra $\mathcal{A}_{\infty}$. An analogous work was made in [IK99] for the case of $Z\left(\mathbb{C}\left[\mathcal{S}_{n}\right]\right)$.

From the formula of the product of basis elements in $\mathcal{A}_{\infty}$, given in Corollary 3.16, we see that the function

$$
\operatorname{deg}_{1}\left(T_{\lambda}\right)=|\lambda|
$$

defines a filtration on $\mathcal{A}_{\infty}$.

In order to obtain other filtrations on $\mathcal{A}_{\infty}$, we give a decomposition of any partial bijection into partial bijections with coset-type equal to (1) or (2). We call cycle of length $r+1$ a partial bijection with coset-type equal to $(r+1)$, where $r$ is a positive integer. We write a cycle $\mathcal{C}$ of length $r+1$ as follows (see [AC12, page 2480]):

$$
\mathcal{C}=\left(c_{1}, c_{2}: c_{3}, c_{4}: \cdots: c_{4 r+1}, c_{4 r+2}: c_{4 r+3}, c_{4 r+4}\right) .
$$

This means that:

$$
\begin{array}{lr}
\left\{c_{2 i+1}, c_{2 i+2}\right\}=\rho\left(k_{i}\right) \text { for some } k_{i} & i=0, \cdots, 2 r+1 \\
\mathcal{C}\left(c_{1}\right)=c_{4 r+4} & \\
\mathcal{C}\left(c_{4 l+1}\right)=c_{4 l} & l=1, \cdots, r \\
\mathcal{C}\left(c_{4 l+2}\right)=c_{4 l+3} & l=0, \cdots, r
\end{array}
$$

Example 6.1. With this notation, the longest cycle in Figure 1, which we draw here again for convenience,

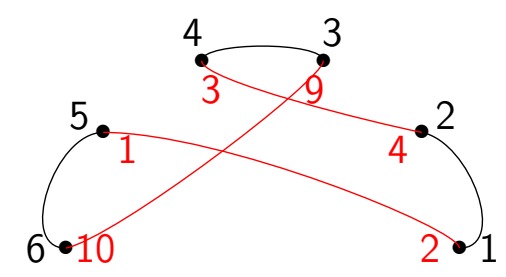

may be written as:

$$
(1,2: 4,3: 4,3: 9,10: 6,5: 1,2)
$$

Notation. For a partial bijection $\alpha$ and $x \in \mathcal{D}_{n}$, we write $\alpha \in x$ to say that the coefficient of $\alpha$ in $x$ is non-zero.

Observation 6.1. Let $\alpha, \beta$ and $\gamma$ be three partial bijections such that $\gamma \in \alpha * \beta$. If $\alpha \in x$ and $\beta \in y$ where $x$ and $y$ are two elements of $\mathcal{D}_{n}$ with non-negative coefficients, then $\gamma \in x * y$.

Lemma 6.1 . For any cycle $\mathcal{C}$ of length $r+1$, there exist $r$ partial bijections $\tau_{1}, \cdots, \tau_{r}$ with coset-type (2), such that $\mathcal{C} \in \tau_{1} * \cdots * \tau_{r}$. 
Proof. Let $\mathcal{C}$ be a cycle of length $r+1$ written in generic form as in (24). We can check that $\mathcal{C}$ can be written as follows:

$$
\mathcal{C}=\mathcal{K} \circ \mathcal{J}
$$

where

$$
\begin{aligned}
\mathcal{K}=\left(c_{3}, c_{4}: c_{4}, c_{3}\right) \cdots & \left(c_{4 r-5}, c_{4 r-4}: c_{4 r-4}, c_{4 r-5}\right) \\
& \left(c_{4 r-1}, c_{4 r}: c_{4 r+4}, c_{4 r+3}: c_{4 r+3}, c_{4 r+4}: c_{4 r}, c_{4 r-1}\right)
\end{aligned}
$$

and

$$
\mathcal{J}=\left(c_{1}, c_{2}: c_{3}, c_{4}: \cdots: c_{4 r-3}, c_{4 r-2}: c_{4 r-1}, c_{4 r}\right)\left(c_{4 r+1}, c_{4 r+2}: c_{4 r+3}, c_{4 r+4}\right) .
$$

Then, if we denote by $\tau_{1}$ the cycle of length 2 of $\mathcal{K}$ and by $\mathcal{C}_{1}$ the cycle of length $r$ of $\mathcal{J}$, we have:

$$
\mathcal{C} \in \tau_{1} * \mathcal{C}_{1}, \operatorname{ct}\left(\tau_{1}\right)=(2) \text { and } \operatorname{ct}\left(\mathcal{C}_{1}\right)=(r)
$$

In the same way we can write:

$$
\mathcal{C}_{1} \in \tau_{2} * \mathcal{C}_{2} \text { with } \operatorname{ct}\left(\tau_{2}\right)=(2) \text { and } \operatorname{ct}\left(\mathcal{C}_{2}\right)=(r-1) \text {. }
$$

Using the observation above, we get:

$$
\mathcal{C} \in \tau_{1} * \tau_{2} * \mathcal{C}_{2}
$$

Thus, by iteration we obtain:

$$
\mathcal{C} \in \tau_{1} * \tau_{2} * \cdots * \tau_{r} \text { with } \operatorname{ct}\left(\tau_{i}\right)=(2) \text { for all } i=1, \cdots, r \text {. }
$$

This proves the lemma.

Lemma 6.2. For any partial bijection $\tau$ with coset-type $\rho=\left(\rho_{1}, \cdots, \rho_{l}\right)$, there exist $r$ partial bijections $\tau_{1}, \cdots, \tau_{r}$ with coset-type $(2)$ and $m_{1}(\rho)$ partial bijections $\beta_{1}, \cdots, \beta_{m_{1}(\rho)}$ with coset-type (1) such that:

$$
\tau \in \tau_{1} * \cdots * \tau_{r} * \beta_{1} * \cdots * \beta_{m_{1}(\rho)}
$$

where $r=|\rho|-m_{1}(\rho)$.

Proof. Every cycle in the graph $\Gamma(\tau)$ associated to $\tau$ can be seen as a partial bijection on its own. Let $\beta_{1}, \cdots, \beta_{m_{1}(\rho)}$ the $m_{1}(\rho)$ partial bijections corresponding to the cycles of length 1 of $\tau$. The other $m(\rho)=l(\rho)-m_{1}(\rho)$ partial bijections corresponding to the cycles of $\tau$ with length greater than 1 are denoted by $\alpha_{1}, \cdots, \alpha_{m(\rho)}$. The length of $\alpha_{i}$ is $\rho_{i}$. By Lemma 6.1, for every $\alpha_{i}$ we can write:

$$
\alpha_{i} \in \tau_{1}^{i} * \cdots * \tau_{\rho_{i}-1}^{i} \text { with } \operatorname{ct}\left(\tau_{j}^{i}\right)=(2) \text { for } j=1, \cdots, \rho_{i}-1 \text {. }
$$


Then, since

$$
\tau \in \alpha_{1} * \cdots * \alpha_{m(\rho)} * \beta_{1} * \cdots * \beta_{m_{1}(\rho)}
$$

we can write:

$$
\tau \in \tau_{1}^{1} * \cdots * \tau_{\rho_{1}-1}^{1} * \cdots * \tau_{1}^{m(\rho)} * \cdots * \tau_{\rho_{m(\rho)}-1}^{m(\rho)} * \beta_{1} * \cdots * \beta_{m_{1}(\rho)} .
$$

The number of $\tau$ 's that appear in this decomposition is equal to $\left(\rho_{1}-1\right)+\cdots+\left(\rho_{m(\rho)}-1\right)=$ $|\rho|-m_{1}(\rho)-l(\rho)+m_{1}(\rho)=|\rho|-l(\rho)$, which proves the lemma.

For a partial bijection $\tau$ with coset-type $\rho$, we define the following functions:

$$
\begin{aligned}
& \operatorname{deg}_{2}(\tau)=|\rho|-l(\rho) \\
& \operatorname{deg}_{3}(\tau)=|\rho|-l(\rho)+m_{1}(\rho) .
\end{aligned}
$$

Consider the decomposition of $\tau$ given in Proposition 6.2. We have:

$$
\begin{aligned}
& \operatorname{deg}_{2}\left(\tau_{i}\right)=1 \\
& \operatorname{deg}_{2}\left(\beta_{i}\right)=0, \\
& \operatorname{deg}_{3}\left(\tau_{i}\right)=1 \\
& \operatorname{deg}_{3}\left(\beta_{i}\right)=1
\end{aligned}
$$

Thus

$$
\operatorname{deg}_{2}(\tau)=|\rho|-l(\rho)=\operatorname{deg}_{2}\left(\tau_{1}\right)+\cdots+\operatorname{deg}_{2}\left(\tau_{r}\right)+\operatorname{deg}_{2}\left(\beta_{1}\right)+\cdots+\operatorname{deg}_{2}\left(\beta_{m_{1}(\rho)}\right),
$$

and

$$
\operatorname{deg}_{3}(\tau)=|\rho|-l(\rho)+m_{1}(\rho)=\operatorname{deg}_{3}\left(\tau_{1}\right)+\cdots+\operatorname{deg}_{3}\left(\tau_{r}\right)+\operatorname{deg}_{3}\left(\beta_{1}\right)+\cdots+\operatorname{deg}_{3}\left(\beta_{m_{1}(\rho)}\right) .
$$

Proposition 6.3. The functions

$$
\begin{aligned}
& \operatorname{deg}_{2}\left(T_{\rho}\right)=|\rho|-l(\rho), \\
& \operatorname{deg}_{3}\left(T_{\rho}\right)=|\rho|-l(\rho)+m_{1}(\rho),
\end{aligned}
$$

define two filtrations on $\mathcal{A}_{\infty}$.

Proof. Let $\sigma$ and $\tau$ be two partial bijections with coset-type $\lambda$ and $\rho$. We want to show that:

$$
\operatorname{deg}_{i}(\sigma * \tau) \leqslant \operatorname{deg}_{i}(\sigma)+\operatorname{deg}_{i}(\tau), \quad \text { for } i=1,2,
$$

where $\operatorname{deg}_{i}(\sigma * \tau)=\max _{\alpha \in \sigma * \tau} \operatorname{deg}_{i}(\alpha)$.

Because of Proposition 6.2, $\tau$ can be decomposed into cycles of lengths 2 and 1 . In other 
words, there exist $r=|\rho|-m_{1}(\rho)$ partial bijections $\tau_{1}, \cdots, \tau_{r}$ with coset-type $(2)$ and $m_{1}(\rho)$ partial bijections $\beta_{1}, \cdots, \beta_{m_{1}(\rho)}$ with coset-type (1) such that:

$$
\tau \in \tau_{1} * \cdots * \tau_{r} * \beta_{1} * \cdots * \beta_{m_{1}(\rho)} .
$$

For any partial bijection $\alpha$ such that $\alpha \in \sigma * \tau$, we have $\alpha \in \sigma * \tau_{1} * \cdots * \tau_{r} * \beta_{1} * \cdots * \beta_{m_{1}(\rho)}$ by Observation 6.1 since $\tau \in \tau_{1} * \cdots * \tau_{r} * \beta_{1} * \cdots * \beta_{m_{1}(\rho)}$. Thus,

$$
\operatorname{deg}_{i}(\sigma * \tau) \leqslant \operatorname{deg}_{i}\left(\sigma * \tau_{1} * \cdots * \tau_{r} * \beta_{1} * \cdots * \beta_{m_{1}(\rho)}\right) \quad \text { for } i=1,2 .
$$

If for any cycle $\mathcal{C}$ with length 2 or 1 and for any partial bijection $\theta$ we have,

$$
\operatorname{deg}_{i}(\theta * \mathcal{C}) \leqslant \operatorname{deg}_{i}(\theta)+\operatorname{deg}_{i}(\mathcal{C}), \quad \text { for } i=2,3,
$$

then we can write:

$$
\begin{aligned}
\operatorname{deg}_{i}(\sigma * \tau) & \leqslant \operatorname{deg}_{i}\left(\sigma * \tau_{1} * \cdots * \tau_{r} * \beta_{1} * \cdots * \beta_{m_{1}(\rho)}\right) \\
& \leqslant \operatorname{deg}_{i}\left(\sigma * \tau_{1} * \cdots * \tau_{r} * \beta_{1} * \cdots * \beta_{m_{1}(\rho)-1}\right)+\operatorname{deg}_{i}\left(\beta_{m_{1}(\rho)}\right) \\
& \vdots \\
& \leqslant \operatorname{deg}_{i}(\sigma)+\operatorname{deg}_{i}\left(\tau_{1}\right)+\cdots+\operatorname{deg}_{i}\left(\tau_{r}\right)+\operatorname{deg}_{i}\left(\beta_{1}\right)+\cdots+\operatorname{deg}_{i}\left(\beta_{m_{1}(\rho)}\right) \\
& \leqslant \operatorname{deg}_{i}(\sigma)+\operatorname{deg}_{i}(\tau) .
\end{aligned}
$$

Therefore it is enough to prove the formula (25). Let $\theta$ be a partial bijection with cosettype $\delta$. If

$$
\mathcal{C}=\left(c_{1}, c_{2}: c_{3}, c_{4}\right)
$$

is a cycle of length 1 , we have two cases:

1. If $\left\{c_{3}, c_{4}\right\}$ is in the domain of $\theta$ : In this case the partial bijections that appear in the expansion of the product $\theta * \mathcal{C}$ have the same coset-type as $\theta$ and we have

$$
\operatorname{deg}_{i}(\theta * \mathcal{C}) \leqslant \operatorname{deg}_{i}(\theta)+\operatorname{deg}_{i}(\mathcal{C}), \quad \text { for } i=2,3 .
$$

2. If not, then all the partial bijections that appear in the expansion of the product $\theta * \mathcal{C}$ have the coset-type $\delta \cup(1)$. Then, we can check easily that $\operatorname{deg}_{i}(\theta * \mathcal{C}) \leqslant$ $\operatorname{deg}_{i}(\theta)+\operatorname{deg}_{i}(\mathcal{C})$, for $i=2,3$.

Now, if $\mathcal{C}=\left(c_{1}, c_{2}: c_{3}, c_{4}: c_{5}, c_{6}: c_{7}, c_{8}\right)$ is a cycle of length 2 , the figure of $\mathcal{C}$ is represented on Figure 9. We have 4 cases. We give for each case the general result without the details of the proofs. They simply consist in computing compositions of permutations.

1. $\left\{c_{3}, c_{4}\right\}$ and $\left\{c_{7}, c_{8}\right\}$ do not appear in the domain of $\theta$ :

In this case, the partial bijections that appear in the expansion of the product $\theta * \mathcal{C}$ have coset-type $\delta \cup(2)$. 


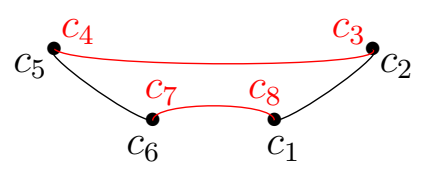

Figure 9: The cycle $\mathcal{C}$.

2. One of the sets $\left\{c_{3}, c_{4}\right\}$ and $\left\{c_{7}, c_{8}\right\}$ (for example $\left\{c_{3}, c_{4}\right\}$ ) appears in the domain of a cycle $\omega$ of $\theta$ and the other does not.

Suppose that $\omega$ is as represented on the left side of Figure 10. Then, a cycle with the form drawn on the right side of Figure 10 appears in the expansion of the product $\theta * \mathcal{C}$. Note that some exterior labels are missing in this figure. To explain this, let us recall that the product $\alpha_{1} * \alpha_{2}$ of two partial bijections $\alpha_{1}$ and $\alpha_{2}$ is defined using an average of some partial bijections $\alpha$. When the extremity on an edge have no exterior labels, that means that the elements of any pair $\rho(k)$ different from $\left\{c_{1}, c_{2}\right\}$ and $\left\{c_{5}, c_{6}\right\}$ can be used as labels and that we shall average over all possibilities. We will also use this convention in the last two cases. Thus, in this case, the coset-type of each partial bijection that appears in the expansion of the product $\theta * \mathcal{C}$ has the same number of parts as $\delta$ and its size is equal to $|\delta|+1$.
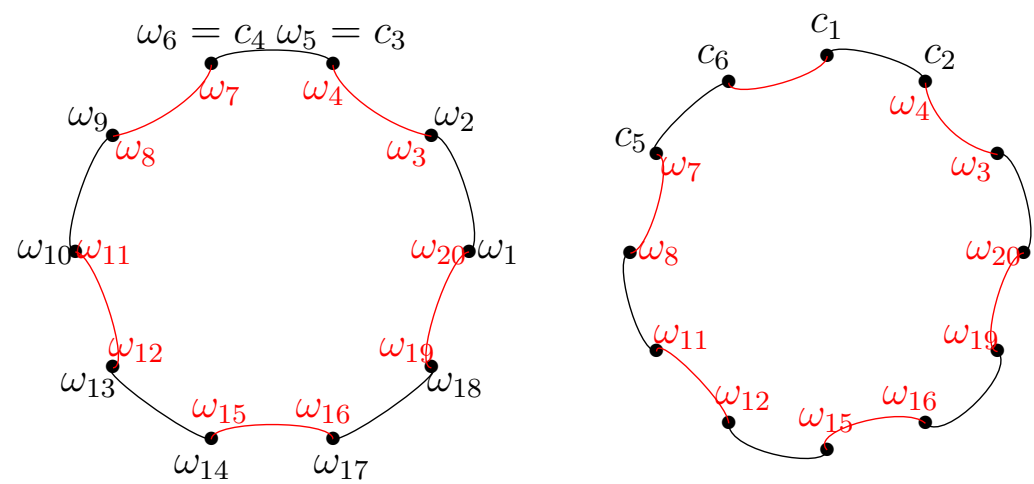

Figure 10: The cycle $\omega$ of $\theta$ and the form of the cycle that appears in $\theta * \mathcal{C}$.

3. Both sets $\left\{c_{3}, c_{4}\right\}$ and $\left\{c_{7}, c_{8}\right\}$ appear in the domain of the same cycle $\omega$ of $\theta$.

Consider the exterior labels of this cycle $\omega$. Among them there are $c_{3}, c_{4}, c_{7}$ and $c_{8}$ and we know that $c_{3}$ and $c_{4}$ (resp. $c_{7}$ and $c_{8}$ ) appear consecutively. Then there are two cases that shall be considered separately. Either labels appear in cyclic order $c_{3}, c_{4}, \cdots, c_{7}, c_{8}$ or $c_{3}, c_{4}, \cdots, c_{8}, c_{7}$. These two cases are represented on Figure 11.

Note that, on the right side of Figure 11, labels $c_{7}$ and $c_{8}$ are switched. The forms of cycles that appear in the expansion of the product $\theta * \mathcal{C}$ in each case are given on Figure 12. 

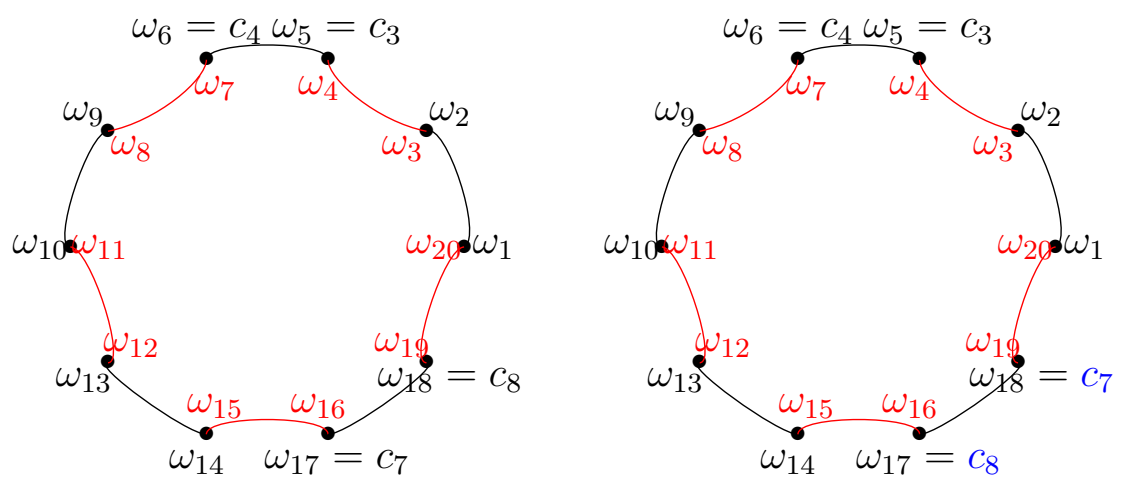

Figure 11: The first possible form of $\omega$ (left side) and the second one.

Thus, in the first case the cycle is cut into two cycles, then the coset-type of each partial bijection that appears in the expansion of the product $\theta * \mathcal{C}$ has the same size as $\delta$ and $l(\delta)+1$ parts. While in the second nothing changes and the coset-type of each partial bijection that appears in the expansion of the product $\theta * \mathcal{C}$ has the same size as $\delta$ and the same number of parts.
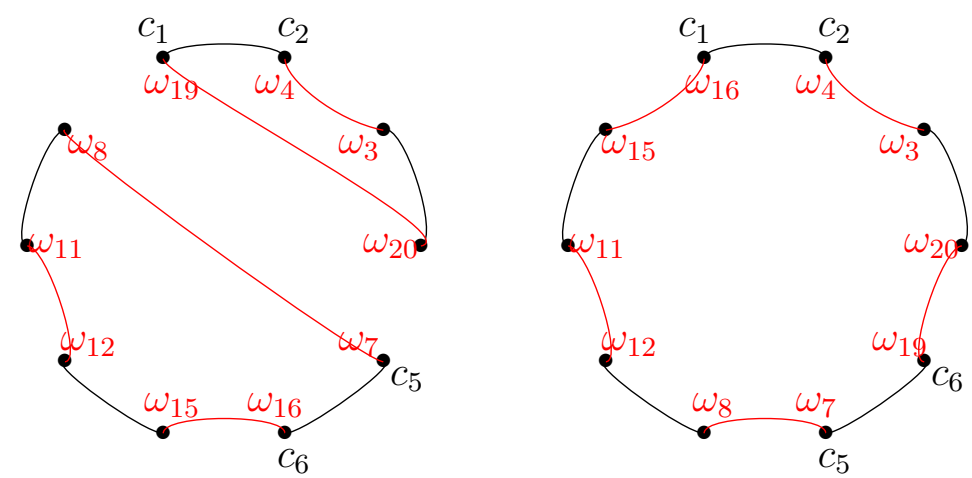

Figure 12: Cycles in $\theta * \mathcal{C}$ corresponding to first and second forms of $\omega$.

4. The two sets $\left\{c_{3}, c_{4}\right\}$ and $\left\{c_{7}, c_{8}\right\}$ appear in the domain of two different cycles of $\theta$. For example we take the two cycles represented on Figure 13. Then a cycle with the form drawn on Figure 14 appears in the expansion of the product $\theta * \mathcal{C}$. In this case the two cycles are joined to form a cycle, thus the coset-type of each partial bijection that appears in the expansion of the product $\theta * \mathcal{C}$ has the same size as $\delta$ and $l(\delta)-1$ parts. 

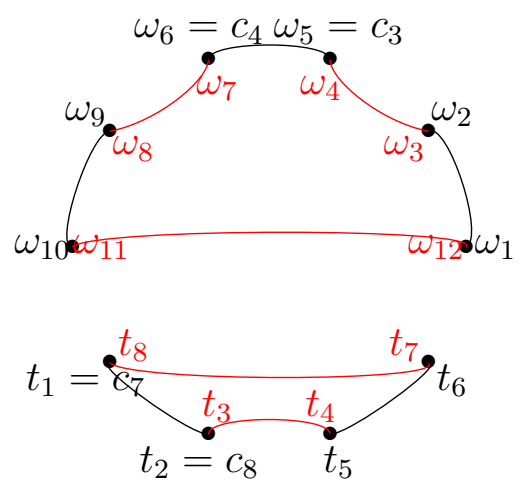

Figure 13: The two cycles.

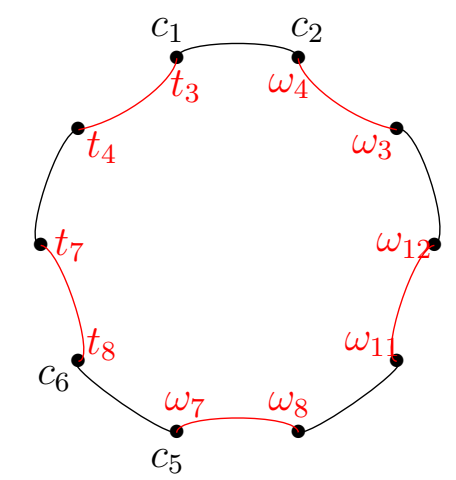

Figure 14: The two cycles are joined.

In these four cases, we can check that we have $\operatorname{deg}_{i}(\theta * \mathcal{C}) \leqslant \operatorname{deg}_{i}(\theta)+\operatorname{deg}_{i}(\mathcal{C})$, for $i=2,3$ and this ends the proof of Proposition 6.3.

These filtrations allow us to get upper bounds for the degree of the polynomials $f_{\lambda \delta}^{\rho}(n)$ that appear in Theorem 2.1.

Proposition 6.4. Let $\lambda, \delta$ and $\rho$ be three proper partitions, the degree of $f_{\lambda \delta}^{\rho}(n)$ satisfies:

$$
\begin{aligned}
& \operatorname{deg}\left(f_{\lambda \delta}^{\rho}(n)\right) \leqslant|\lambda|+|\delta|-|\rho|, \\
& \operatorname{deg}\left(f_{\lambda \delta}^{\rho}(n)\right) \leqslant|\lambda|+|\delta|-|\rho|-l(\lambda)-l(\delta)+l(\rho) .
\end{aligned}
$$

Proof. From the proof of Theorem 2.1, the degree of $f_{\lambda \delta}^{\rho}(n)$ is as follows:

$$
\operatorname{deg}\left(f_{\lambda \delta}^{\rho}(n)\right)=\max _{b_{\lambda \delta}^{\rho \cup\left(1^{j}\right)} \neq 0} j
$$


On the other hand, since $\operatorname{deg}_{i}$ is a filtration for $i=1,2,3$, we obtain the following inequality:

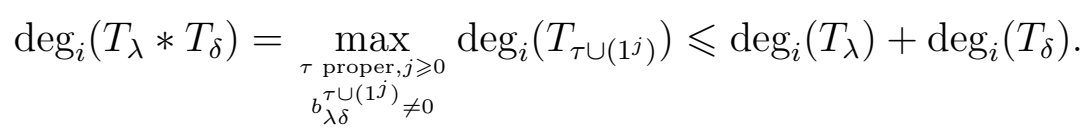

Then we get the three following inequalities corresponding to the three filtrations $\operatorname{deg}_{1}$, $\operatorname{deg}_{2}$ and $\operatorname{deg}_{3}$ :

$$
\begin{aligned}
& \max _{\substack{\tau \text { proper }, j \geqslant 0 \\
b_{\lambda \delta}^{\tau \cup\left(1^{j}\right)} \neq 0}}|\tau|+j \leqslant|\lambda|+|\delta|, \\
& \max _{\substack{\tau \text { proper }, j \geqslant 0 \\
b_{\lambda \delta}^{\tau \cup(1 j)} \neq 0}}|\tau|+j-l(\tau)-j \leqslant|\lambda|-l(\lambda)+|\delta|-l(\delta), \\
& \max _{\substack{\tau \text { proper }, j \geqslant 0 \\
b_{\lambda \delta}^{\tau \cup\left(1^{j}\right)} \neq 0}}|\tau|+j-l(\tau)-j+j \leqslant|\lambda|-l(\lambda)+|\delta|-l(\delta) .
\end{aligned}
$$

The second inequality does not give any information about the degree of $f_{\lambda \delta}^{\rho}(n)$, while using the first and the third inequality with the fact that:

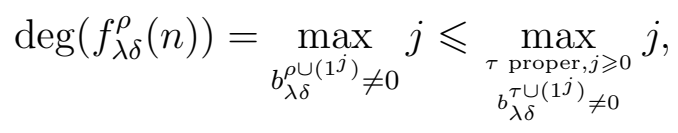

we get directly the result.

\section{Acknowledgement}

I would like to thank my advisors Jean-Christophe Aval and Valentin Féray for helpful discussions and suggestions and enlightening ideas of some proofs. I am also deeply grateful to them for reading and improving this article several times.

\section{References}

[AC12] Kürşat Aker and Mahir Bilen Can. Generators of the hecke algebra of $\left(S_{2 n}, H_{n}\right)$. Advances in Mathematics, 231(5):2465 - 2483, 2012.

[BÖ14] M. Bilen Can and Ş. Özden. Corrigendum to "Generators of the Hecke algebra of $\left(S_{2 n}, B_{n}\right)$ ". To appear in Advances in Mathematics, arXiv:1407.3700, 2014.

[Can13] Mahir Bilen Can. Personal communication. 2013.

[Cor75] R. Cori. Un code pour les graphes planaires et ses applications. Number 27 in Astérisque. Société Mathématique de France, 1975. 169 pages.

[DF14] Maciej Dołęga and Valentin Féray. Gaussian fluctuations of young diagrams and structure constants of jack characters. arXiv:1402.4615, 2014. 
[FH59] H. Farahat and G. Higman. The centres of symmetric group rings. Proc. Roy. Soc. (A), 250:212-221, 1959.

[GJ96] I. P. Goulden and D. M. Jackson. Maps in locally orientable surfaces, the double coset algebra, and zonal polynomials. Can. J. Math., 48(3):569-584, 1996.

[GS98] A. Goupil and G. Schaeffer. Factoring n-cycles and counting maps of given genus. Eur. J. Comb., 19(7):819-834, 1998 .

[IK99] V. Ivanov and S. Kerov. The algebra of conjugacy classes in symmetric groups, and partial permutations. Zap. Nauchn. Sem. S.-Peterburg. Otdel. Mat. Inst. Steklov. (POMI), 256(3):95-120, 1999.

[Jam61] Alan T. James. Zonal polynomials of the real positive definite symmetric matrices. Annals of Mathematics, 74(3):456-469, 1961.

[JV90] D.M. Jackson and T.I. Visentin. A character theoretic approach to embeddings of rooted maps in an orientable surface of given genus. Trans. AMS, 322:343-363, 1990.

[Las08] M. Lassalle. A positivity conjecture for Jack polynomials. Math. Res. Lett., 15(4):661-681, 2008 .

[Mac95] I.G. Macdonald. Symmetric functions and Hall polynomials. Oxford Univ. Press, second edition, 1995

[Mél13] Pierre-Loïc Méliot. Partial isomorphisms over finite fields. Journal of Algebraic Combinatorics, pages 1-54, 2013.

[OO97] Andrei Okounkov and Grigori Olshanski. Shifted Jack polynomials, binomial formula, and applications. Mathematical Research Letters, 4:69-78, 1997.

[Sag] Sage mathematical software, version 4.8, http://www . sagemath.org.

[Sol90] Louis Solomon. The bruhat decomposition, tits system and iwahori ring for the monoid of matrices over a finite field. Geometriae Dedicata, 36(1):15-49, 1990. 